\title{
p53-directed translational control can shape and expand the universe of $p 53$ target genes
}

\author{
S Zaccara ${ }^{1}$, T Tebaldi $^{2}$, C Pederiva ${ }^{1}$, Y Ciribilli ${ }^{1}$, A Bisio ${ }^{1}$ and A Inga ${ }^{*, 1}$
}

The increasing number of genome-wide transcriptome analyses focusing on p53-induced cellular responses in many cellular contexts keeps adding to the already numerous p53-regulated transcriptional networks. To investigate post-transcriptional controls as an additional dimension of p53-directed gene expression responses, we performed a translatome analysis through polysomal profiling on MCF7 cells upon 16 hours of doxorubicin or nutlin-3a treatment. The comparison between the transcriptome and the translatome revealed a considerable level of uncoupling, characterized by genes whose transcription variations did not correlate with translation variations. Interestingly, uncoupled genes were associated with apoptosis, DNA and RNA metabolism and cell cycle functions, suggesting that post-transcriptional control can modulate classical p53-regulated responses. Furthermore, even for well-established p53 targets that were differentially expressed both at the transcriptional and translational levels, quantitative differences between the transcriptome, subpolysomal and polysomal RNAs were evident. As we searched mechanisms underlying gene expression uncoupling, we identified the p53-dependent modulation of six RNA-binding proteins, where hnRNPD (AUF1) and CPEB4 are direct p53 transcriptional targets, whereas SRSF1, DDX17, YBX1 and TARDBP are indirect targets (genes modulated preferentially in the subpolysomal or polysomal mRNA level) modulated at the translational level in a p53-dependent manner. In particular, YBX1 translation appeared to be reduced by p53 via two different mechanisms, one related to $\mathrm{mTOR}$ inhibition and the other to miR-34a expression. Overall, we established p53 as a master regulator of translational control and identified new p53-regulated genes affecting translation that can contribute to p53-dependent cellular responses. Cell Death and Differentiation (2014) 21, 1522-1534; doi:10.1038/cdd.2014.79; published online 13 June 2014

Discovered nearly 35 years ago, tumor suppressor p53, which is often described as the 'guardian of the genome,' acts prominently as a transcription factor in many biological processes including DNA metabolism, apoptosis and cell cycle regulation. ${ }^{1}$ Although the role of p53 is generally considered to be at the level of transactivation via binding to target sequences, there are several other ways by which it can determine its cellular responses including, for example, interaction with other transcription factors. ${ }^{2}$

Post-transcriptional and translational controls provide fine tuning of transcriptional outcomes in eukaryotic somatic cells. ${ }^{3}$ More than $90 \%$ of all coding transcripts appear to be subject to this regulation, especially at translation initiation, ${ }^{4}$ considered as the rate-limiting step of the whole process. ${ }^{3,5}$ By binding mainly to the $5^{\prime}$ and $3^{\prime}$ untranslated regions (5'UTR; 3'UTR) of mRNAs, miRNAs-other non-coding RNAs and RNA-binding proteins (RBPs) - were shown to participate in the regulation of translation. ${ }^{6}$ An unexpected complexity in the modulation of the fate of mRNAs along with a widespread alteration of that process in cancer cells was found in recent studies. ${ }^{7-9}$ The synthesis of the 053 protein itself has been shown to be modulated by miR-125 $b^{10}$ or by RPL26 and nucleolin, that produce opposite effects on the rate of p53 mRNA translation. ${ }^{11}$ Moreover, p53 target genes, including CDKN1A (p21), BBC3 (PUMA) and BAX, can be regulated post-transcriptionally by miRNAs or RBPs, some of which can be direct p53 target genes. The impact that this additional level of regulation can have on the p53 response networks has been recently reviewed. ${ }^{12-17}$

All these mechanisms to control the fate of mRNAs may account for the lack of correlation-referred to as uncouplingbetween relative changes in the total cellular mRNA levels (corresponding to the transcriptome (transcripts examined from total RNA extractions)) and protein abundances (the proteome) after p53 activation. ${ }^{18}$ To investigate p53-dependent uncoupling at the genome level, we compared the transcriptome after doxorubicin (Doxo) or nutlin-3a (Nutlin) treatment with the translatome (transcript examined from polysomal mRNA extractions, considered as actively translated), analyzed by polysomal profiling, a technique that allows quantification of mRNAs associated with the polysomes as a proxy for the proteome. ${ }^{19}$

Overall, we identified an evident translation selectivity that we considered to be an additional dimension by which p53 can tailor its gene response network.

\section{Results}

Coupled differentially expressed genes after doxorubicin and nutlin-3a treatments are enriched for p53 targets. To characterize the impact of post-transcriptional

\footnotetext{
${ }^{1}$ Laboratory of Transcriptional Networks, Centre for Integrative Biology, CIBIO, University of Trento, Trento, Italy and ${ }^{2}$ Laboratory of Translational Genomics, Centre for Integrative Biology, CIBIO, University of Trento, Trento, Italy

*Corresponding author: A Inga, Laboratory of Transcriptional Networks, Centre for Integrative Biology, CIBIO, University of Trento, via delle Regole 101, Mattarello, Trento 38123, Italy. Tel: + 390461 283099/283714; Fax: + 390461 283091; E-mail: inga @ science.unitn.it

Abbreviations: Tot, total RNA; sub, subpolysomal RNA; pol, polysomal RNA; up, upregulated; down, downregulated; RBPs, RNA-binding proteins; Doxo, doxorubicin; Nutlin, nutlin-3a; DEGs, differentially expressed genes

Received 17.10.13; revised 23.4.14; accepted 30.4.14; Edited by M Oren; published online 13.6.14
} 
regulation in shaping the p53-dependent gene response, we combined polysomal profiling with microarray analysis on MCF7vector cells ${ }^{20}$ (containing wild-type p53) upon doxorubicin (Doxo, $1.5 \mu \mathrm{M}$ ) or nutlin-3a (Nutlin, $10 \mu \mathrm{M}$ ) treatment for $16 \mathrm{~h}$. Both treatments resulted in high p53 induction and similar low levels of toxicity (Supplementary Figure S1). Experiments were conducted also on MCFshp53 cells, which express an shRNA targeting p53. ${ }^{20}$ Residual p53 expression was detected in MCF7shp53 cells, but p21 was not induced by either Doxo or Nutlin (Supplementary Figure S1C).

No significant effects of the treatments on overall polysomal distributions were evident (Figure 1a). For the microarray analysis, we collected subpolysomal ('sub') and polysomal ('pol') mRNA fractions so as to analyze mRNAs that are not actively translated separately from those that are in active translation. ${ }^{21}$ Total mRNA ('tot') was also collected to quantify transcriptome changes (see Materials and Methods).

When we measured the global overlap between expression changes of 'tot' (transcriptome) and 'pol' (translatome), we obtained Spearman correlations of 0.65 and 0.67 after Doxo and Nutlin treatment, respectively (Figure 1b). Hence, many genes exhibited homodirectional changes both in terms of transcriptome and translatome (defined as coupled differentially expressed genes (DEGs)). In the three RNA preparations (tot, pol and sub), we found 239 and 155 commonly upregulated coupled DEGs (DEGs with homodirectional expression changes in transcriptome and translatome) after Doxo and Nutlin treatment and 216 and 301 commonly repressed coupled DEGs (Figure 1c, green, overlapping areas). Among them, we counted 107 upregulated coupled and 118 downregulated-coupled DEGs in both Doxo and Nutlin treatment. (Supplementary Table S1A). A p53 pathway signature was revealed by ingenuity pathway analysis for Doxo and Nutlin upregulated and downregulated coupled DEGs (Figure 1d). Sixty-four out of 225 genes had expression changes consistent with p53 activation ( $P$-value: $\left.7.5^{-39}\right)$ (Supplementary Table S2A). Moreover, ingenuity pathway analysis identified $\mathrm{p} 53$ as the main upstream regulator especially among coupled DEGs (Supplementary Table S3). Interestingly, gene ontology enrichment did not reveal differences between Doxo and Nutlin DEGs (Supplementary Table S2B), consistent with the similarity in cell phenotypes observed at the doses and time point used (Supplementary Figure S1).

Three well-established p53 target genes (MDM2, p21 and PUMA) were validated as coupled DEGs by quantitative PCR (qPCR), whose sensitivity is higher than the microarray's (Figure 1e). Given the significantly higher fold change in the pol fraction compared with the subpolysomal fraction, particularly after Nutlin treatment, p21 and MDM2 translation appeared to be enhanced. We defined this finding a 'translational thrust'. An $8 \mathrm{~h}$ treatment time point was added for comparison (Figure 1e); (Supplementary Figure S2). At the earlier time point the induction of the three p53 target genes, and particularly MDM2, was more robust in response to Nutlin than to Doxo. The PUMA transcript can also be classified as a thrust gene after $8 \mathrm{~h}$ of Nutlin treatment. However, at the $16 \mathrm{~h}$ time point PUMA-relative expression changes were higher in total RNA compared with sub and pol, suggesting that, unlike MDM2 and p21, the PUMA transcript could be subject to an opposite regulation we define here as 'translational drag.' This latter phenomenon could be dependent on several factors, including delayed transactivation, slow pre-mRNA maturation, regulation at the nuclear export level or slow assembly of ribosomes on mRNAs. No evidence of transcriptional or translational changes was seen in treated MCF7shp53 cells (Figure 1e).

The majority of direct p53 target genes are coupled. Nevertheless, we found that expression is uncoupled for about $70 \%$ of DEGs.

The uncoupled, translationally upregulated gene group is enriched for apoptotic functions. Uncoupled DEGs are genes with a major change in relative expression levels compared with mock treatment in only one of the three mRNA preparations: (a) transcriptome (tot); (b) translatome (pol); (c) non-translated subpolysomal mRNAs (sub) (red, blue and yellow circular sectors, respectively, in Figure 1c). We identified 1432 uncoupled DEGs after Doxo and 987 after Nutlin treatment.

First, we focused on uncoupled DEGs that were induced in the translatome but did not change in the transcriptome (Figure 2a); (Supplementary Table S1B). We found 55 translatome-uncoupled DEGs common to the two treatments (Figure 2a). Among them, PHPT1 (14-kDa phosphohistidine phosphatase) ${ }^{22}$ and TP53RK (p53-related protein kinase) ${ }^{23}$ were chosen for validation by qPCR owing to their biological relevance in post-translational control, and because they had not been previously reported as p53-regulated genes. PHPT1 and TP53RK proved to be translational upregulated genes, particularly after $16 \mathrm{~h}$ of treatment (Figure $2 \mathrm{~b}$ ).

We also compared changes in protein levels by western blot analysis (Figure 2c). Considered as a proxy of the proteome, the pol level should reflect the protein level of each transcript. PHPT1 protein levels were slightly induced by both Doxo and Nutlin in MCF7vector cells, whereas no changes occurred in MCF7shp53 (the basal levels were significantly lower) (Figures $2 \mathrm{~b}$ and $\mathrm{c}$ ).

Among induced translatome-uncoupled DEGs, gene ontology analysis revealed enrichment for apoptosis terms after both Doxo and Nutlin treatments (Figure 2d). This observation suggests that modulation of translation efficiency might reinforce the activation of p53-dependent apoptosis, a process that could be important given the generally weaker transcriptional control of p53 target genes in the apoptosis group. ${ }^{24}$ We validated by qPCR TRIAP1, ${ }^{25}$ TRAF4 $^{26}$ and GADD45G ${ }^{27}$ (the full list of genes is presented in Supplementary Figure S3A). According to ChIP-seq data on MCF7 cells, these genes are direct p53 targets. ${ }^{28}$ For all of them, the increase at the polysomal level, especially after Nutlin treatment, was confirmed at both time points. For GADD45G, high level of induction was observed also in the MCF7shp53 cells (Figure 2e).

Overall, we conclude that even if there was a weak modulation at the total mRNA level, p53 or p53-inducing treatments enhanced the translation of these apoptotic genes. 
p53 activation reduced the subpolysomal mRNA levels of DDX17, SRSF1, TARDBP and XRCC2. Relative translation efficiency can decrease by inhibiting translation without impacting mRNA stability, or by destabilizing mRNAs. Given that p53 controls the expression of many miRNAs and RBPs, ${ }^{12}$ it could indirectly impact both mechanisms. DEGs that were selectively downregulated in the sub fraction but did not change in translation might be considered as a
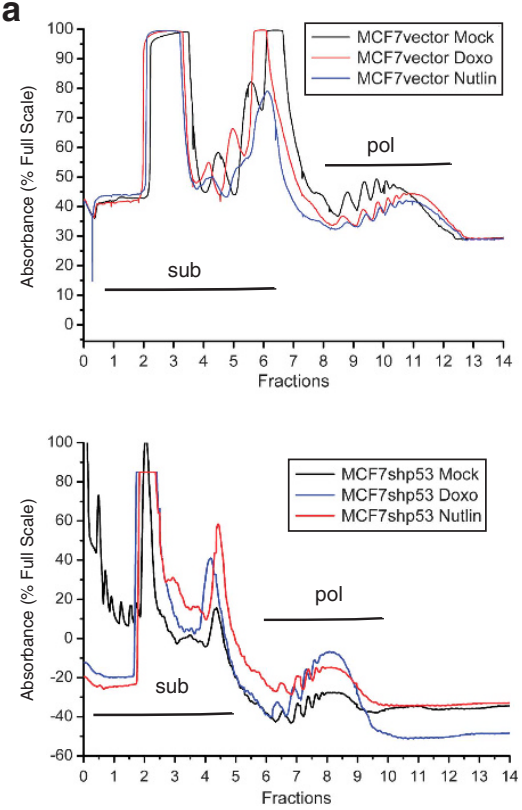

C

Doxo.up

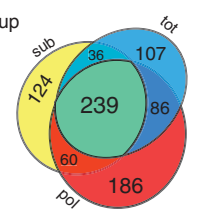

Doxo.down

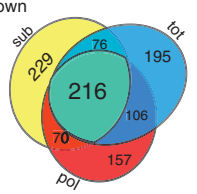

Nutlin.up

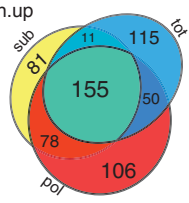

Nutlin.down

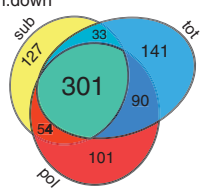

e

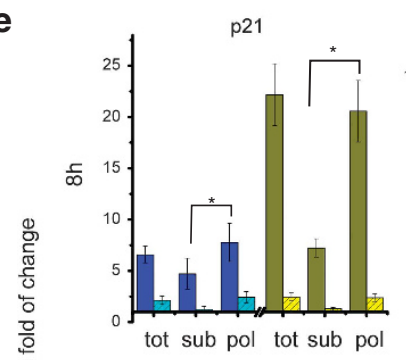

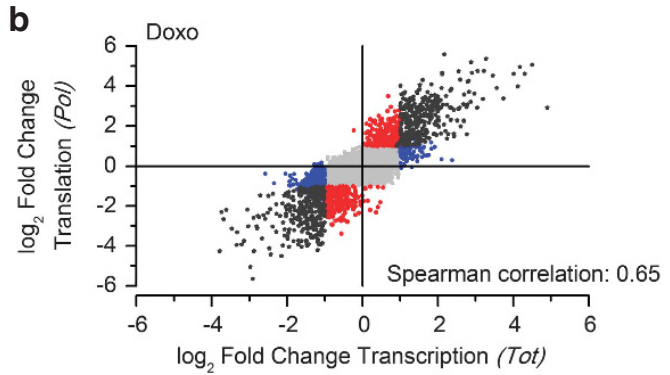

$\log _{2}$ Fold Change Transcription (Tot)

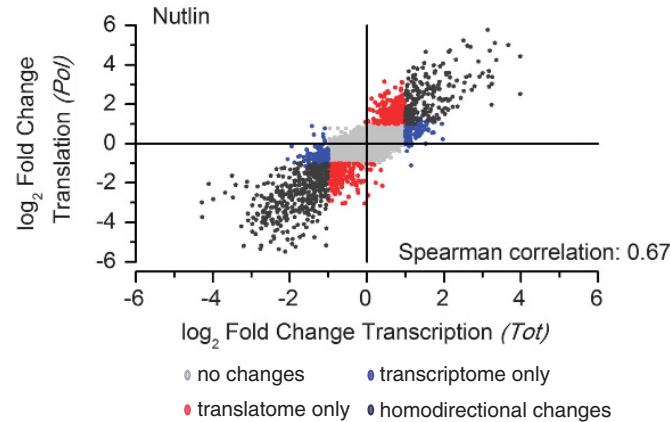

d

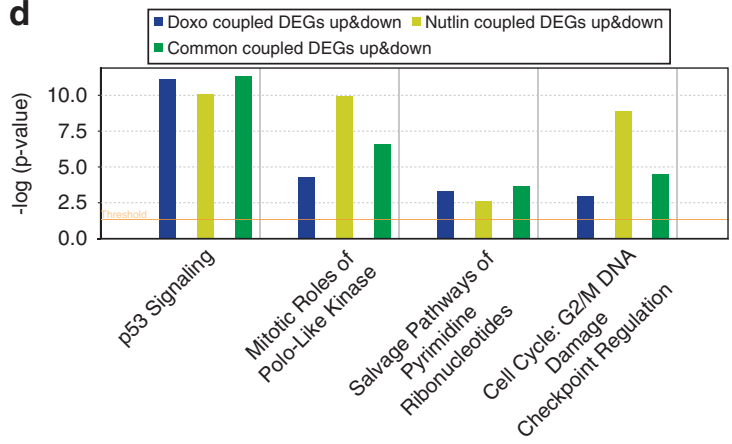

MDM2
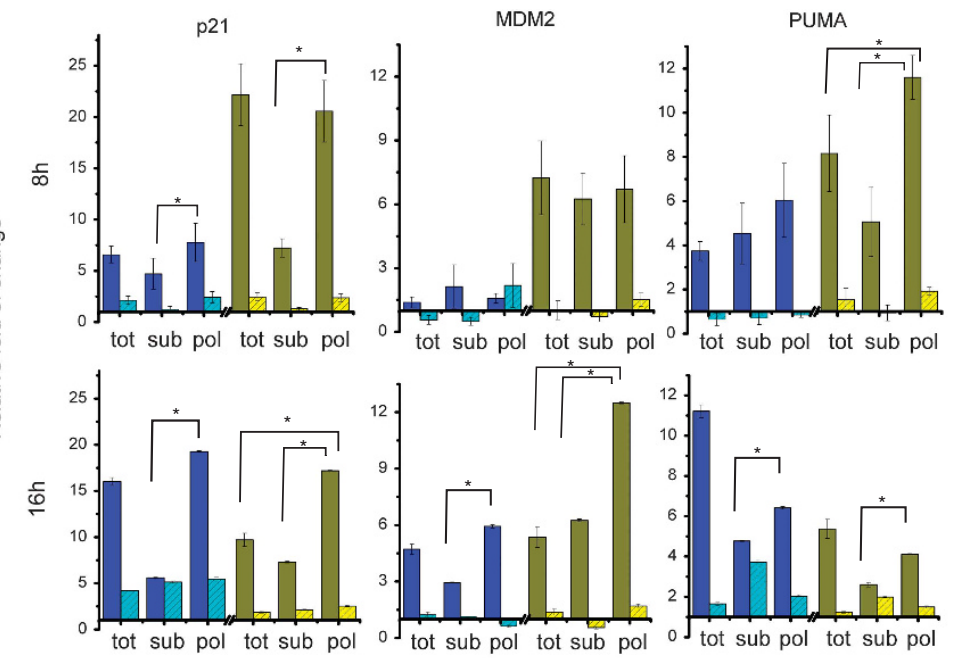

$\square$ MCF7vector Doxo $\square$ MCF7shp53 Doxo $\square$ MCF7vector Nutlin $\square$ MCF7shp53 Nutlin 
candidate targets for a regulation process that targets more selectively mRNA molecules not engaged in translation (Figure 3a). Following Doxo and Nutlin treatments, we observed, respectively, 305 and 160 subpolysomal downregulated DEGs (Supplementary Table S1C) and 81 were common to the two groups. (Figure 3a). 'Mitosis' was the mostly enriched functional category, among which $\mathrm{XRCC}^{29}$ was also validated by qPCR (Figure $3 \mathrm{c}$ ). mRNA-processing categories, such as RNA splicing, were also enriched (Figure 3b); (Supplementary Figure S3B). As we were interested in how p53 might modulate post-transcriptional mechanisms, we also selected three RBPs—SRSF1, DDX17 and TARDBP_for further qPCR validation. DDX17 and SRSF1 mRNAs were confirmed to be downregulated in sub but did not change in tot nor pol after Nutlin treatment in MCF7vector cells. On the contrary, Doxo treatment led to a less-evident down-modulation in the pol fraction that generally was p53-independent (downregulation in the MCF7shp53 cells).

Changes in DDX17 and SRSF1 protein levels were investigated (Figure $3 d$ ). In general, protein levels were more in agreement with their matched pol changes at the $16 \mathrm{~h}$ time point. Moreover, SRSF1 was consistently upregulated in MCF7shp53 at $16 \mathrm{~h}$ post Nutlin treatment, confirming mRNA data and literature reports. ${ }^{30}$

p53 activation leads to translational inhibition of genes involved in mRNA processing and nucleotide binding, including YBX1. Although a reduction in subpolysomal RNA can be interpreted as evidence of reduced mRNA stability, lower polysomal RNA can be a hallmark for decreased translation efficiency of specific mRNAs.

Hence, we examined DEGs that were repressed in polysomal fraction, but did not change in total mRNA (Figure 4a); (Supplementary Table S1D). Although not as first enriched term, gene ontology analysis showed an enrichment for 'mRNA processing' after both treatments (Figure 4b); (Supplementary Figure S3C). DEGs (45) were common to the two treatments (Figure 4a) including five RBPs (YBX1, SNRPA, HNRNPA3, KIAA0020 and DGCR8) among a restricted list (see Materials and Methods).

YBX1 was chosen for validation also because of its reported interaction with $p 53 .{ }^{31}$ Its mRNA was significantly downregulated in polysomal RNA, more than in the total RNA, with a p53-dependent shift from the polysomal to the subpolysomal fraction (Figure 4c). The same trend was observed after $8 \mathrm{~h}$, but only in the Doxo treatment. Moreover, the reduction in pol mRNA corresponds to a reduction in YBX1 protein level (Figure 4d).

The YBX1 transcript was found to have a $5^{\prime}$-terminal oligopyrimidine tract-like mRNA that is suppressed in the polysomal fraction after mTOR inhibition. ${ }^{32}$ Furthermore, p53 can negatively modulate the mTOR pathway via the upregulation of Sestrins (SESN1-2). ${ }^{33}$ SESN1 was among the coupled upregulated DEGs that we identified (Supplementary Table S1A). As apparently p53 could impact on YBX1 mRNA through the mTOR pathway, we examined the impact of Doxo and Nutlin treatments on mTOR activity in comparison with two mechanistically different mTOR inhibitors rapamycin (an allosteric mTORC1 inhibitor) and Torin1 (a selective ATP-competitive mTOR inhibitor) as controls. The amount of p-4EBP1 was reduced by Torin1 and, to a limited extent, by rapamycin, but also by Doxo or Nutlin treatment in MCF7vector cells (Figure 4e). Although YBX1 protein levels were markedly lower after Torin1 and rapamycin treatment independently from p53 status, the reduction was even more evident after both Doxo and Nutlin treatment in MCF7vector cells. This was despite the apparent lower inhibition of mTOR in MCF7shp53 cells, based on p-4EBP1 levels. Collectively, these results suggest an additional mTOR pathwayindependent, p53-dependent mechanism of YBX1 translational regulation.

We searched for published evidence of RBPs or miRNAs that could modulate YBX1 mRNA translation/stability. YBX1 was reported as a target of miR-137 in multidrug-resistant MCF7/ADAM cells, ${ }^{34}$ but we were unable to detect miR-137 in our cell lines, both in treated and untreated conditions. On the basis on a recent CLASH analysis, ${ }^{35}$ miR-34a, a p53-target miRNA, ${ }^{12}$ was found to bind YBX1 $3^{\prime}$ UTR. We confirmed that Doxo and Nutlin increased miR-34a expression only in MCF7vector cells (Supplementary Figure S4A). Moreover, miR-34a ectopic overexpression led to a reduction in YBX1 protein (Figure 4f; Supplementary Figure S4B). Vice versa, upon inhibition of miR-34a, YBX1 levels were slightly increased in the mock condition and were reduced less by Doxo, but not by Nutlin, treatment (Figure 4g; Supplementary Figure S4C).

Figure 1 Differentially expressed genes in the transcriptome and translatome of doxorubicin- and nutlin-3a-treated MCF7 cells. Overall results and coupled differentially expressed genes (DEGs). (a) Profiles after sucrose gradient fractionation of cytoplasmic extracts prepared from MCF7vector (upper plot) and MCF7shp53 (lower plot) cell lines. The conditions tested were mock, doxorubicin (Doxo, $1.5 \mu \mathrm{M}$ ) and nutlin-3a (Nutlin, $10 \mu \mathrm{M}$ ) after $16 \mathrm{~h}$ of treatment. The subpolysomal fractions (sub: free RNA, small$40 \mathrm{~S}$ and large-60S and monosomes-80S) and the polysomal fractions (pol) were separated. Sub and pol fractions (see numbers on $x$-axis) were combined in two separated tubes for RNA extraction. (b) Scatter-plots representing transcriptional and translational $\log _{2}$ fold changes. Spearman correlation was calculated. DEGs in each category (transcriptome only, translatome only and homodirectional changes) are classified according to $\log _{2}$ fold change $>1$ and $<-1$ for induced and repressed genes, respectively, and Benjamini-Hochberg corrected $P$-value $<0.05$. Genes without significant changes are shown in gray. (c) Venn diagrams showing the level of coupling/ uncoupling for upregulated (up) and downregulated (down) DEGs after Doxo and Nutlin treatment. The green overlapping areas represent coupled DEGs that are enriched in all conditions: total RNA (tot), subpolysomal fraction (sub) and polysomal fraction (pol). (d) Coupled DEGs are mainly involved in the p53 pathway. Ingenuity pathways analysis (IPA) was performed on coupled DEGs after Doxo and Nutlin treatment and each of these genes is considered by IPA according to their log fold change and BH corrected $P$-value, integrating up- and downregulated DEGs (up and down). Results on the coupled DEGs that are common to both Doxo and Nutlin treatments are also presented (common coupled DEGs). The $y$-axis displays the significance of the association between DEGs and the canonical pathways measured by Fisher's exact test. A P-value cutoff of 0.05 was used to identify significantly enriched pathways. The plot shows four among the most enriched pathways. (e) Relative expression levels of three established p53 target genes. MCF7vector cells were treated with Doxo and Nutlin for $8 \mathrm{~h}$ (upper plots) or $16 \mathrm{~h}$ (lower plot). MDM2, p21 and PUMA transcript levels were validated by qPCR in total RNA (tot), subpolysomal (sub) and polysomal (pol) fractions as positive controls. To establish p53 dependence, all experiments were conducted also in MCF7shp53 cells. Data are plotted relative to each mock condition and three reference genes. $n=3$. Means \pm S.D. are shown. ${ }^{\star} P<0.05$. The basal expression levels in each RNA fraction are presented in Supplementary Table S7 
a

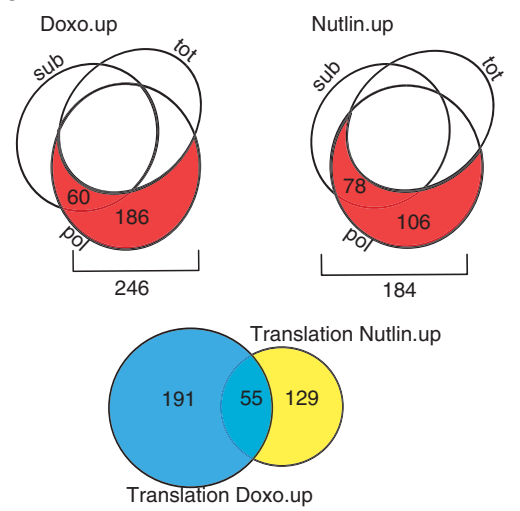

b

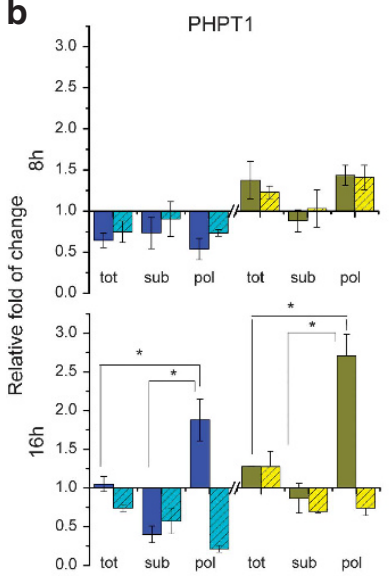

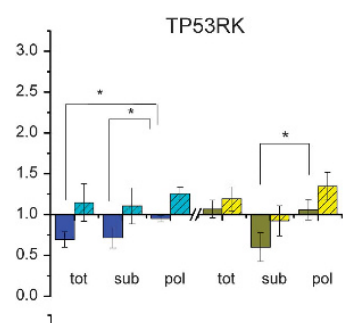

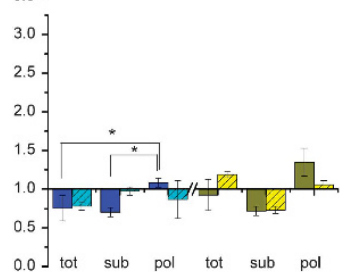

$\square$ MCF7vector Doxo MCF7shp53 Doxo $\square$ MCF7vector Nutlin WIJ MCF7shp53 Nutlin

C
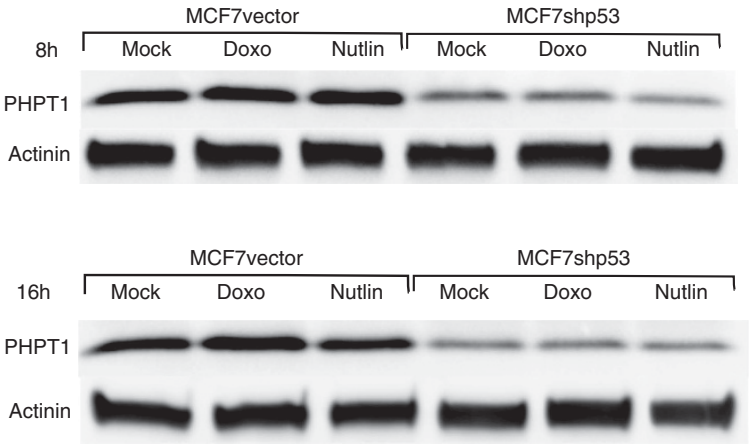

d Polysomal Doxo.up (246)

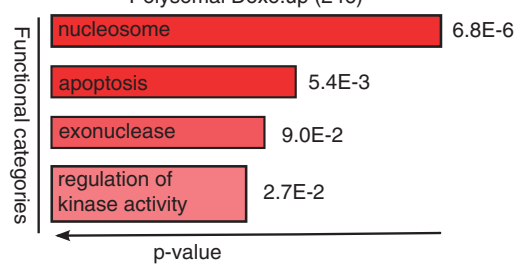

p-value

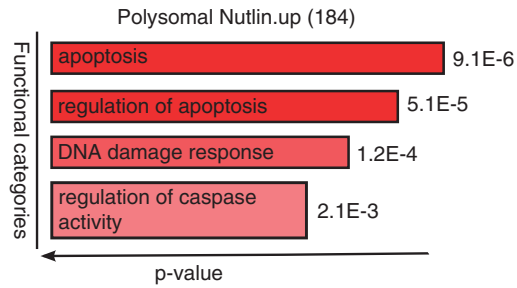

e

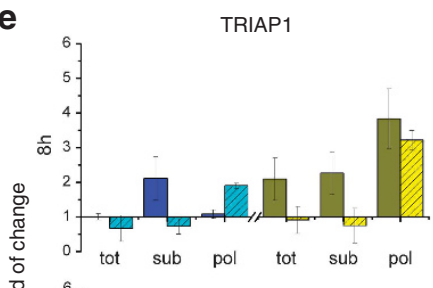

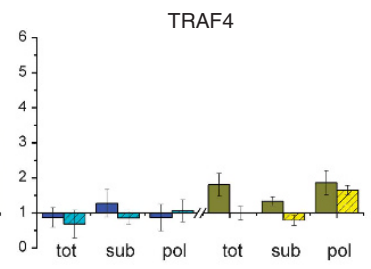
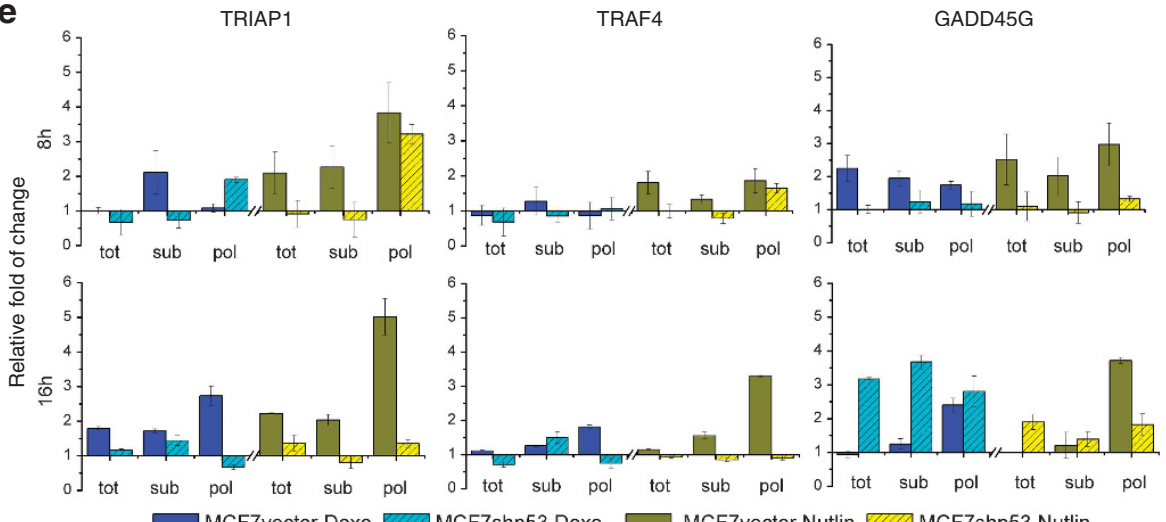

Figure 2 Uncoupled DEGs upregulated only in translation are enriched in apoptosis function. (a) Top: Venn diagrams from Figure 1b, highlighting the uncoupled DEGs that are upregulated in translation after doxorubicin and nutlin-3a treatments. Bottom: Venn diagram showing the level of overlap between DEGs with high translation levels after Doxo and Nutlin treatment. (b) p53 promotes the translation of PHPT1, a modulator of DNA accessibility, and of TP53RK, a putative activator of p53 itself. MCF7vector and MCF7shp53 cell lines were treated with Doxo and Nutlin for $8 \mathrm{~h}$ (upper plots) or $16 \mathrm{~h}$ (lower plots). PHPT1 and TP53RK mRNA levels were validated by qPCR starting from total RNA (tot), subpolysomal (sub) and polysomal (pol) fractions. Data are plotted relative to each mock condition and three reference genes. $n=3$. Means \pm S.D. are shown. ${ }^{*} P<0.05$. (c) Western Blot analysis of PHPT1 protein level in MCF7vector and MCF7shp53 cells after $8 \mathrm{~h}$ (top) and $16 \mathrm{~h}$ (bottom) of treatment with Doxo or Nutlin. Actinin was used as reference protein for loading control. (d) Gene ontology (GO) analysis on upregulated DEGs in the polysomal fraction that did not change in the total RNA. Plots represent the most enriched GO terms obtained by DAVID analysis. P-values of the enriched categories are reported. The analysis was performed on upregulated DEGs after both Doxo and Nutlin treatments. (e) p53 promotes the translation of TRIAP1, TRAF4 and GADD45G, three p53 target genes involved in the apoptotic process. MCF7vector and MCF7shp53 cells were treated with Doxo and Nutlin for $8 \mathrm{~h}$ (upper plots) or $16 \mathrm{~h}$ (lower plots). mRNA levels were measured by qPCR starting from total RNA (tot), subpolysomal (sub) and polysomal (pol) fractions. Data are plotted relative to each mock condition and three reference genes. $n=3$. Means \pm S.D. are shown. ${ }^{*} P<0.05$ 
a

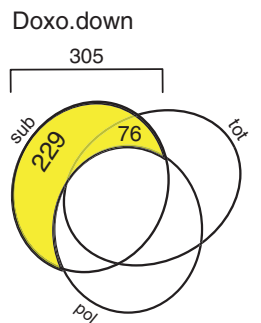

Nutlin.down

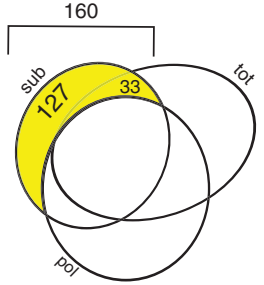

subpolysomal Nutlin.down

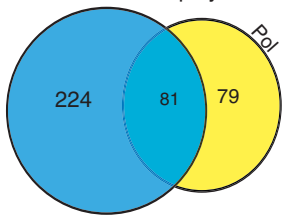

subpolysomal Doxo.down b

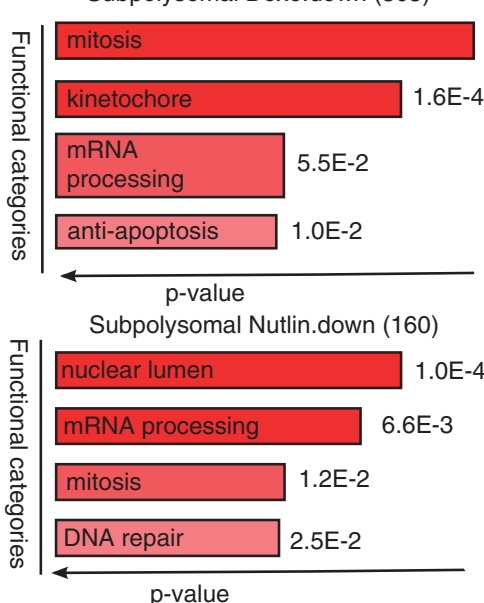

C

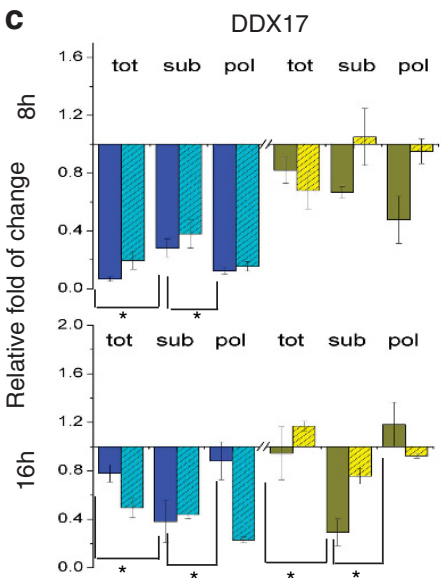

SRSF1

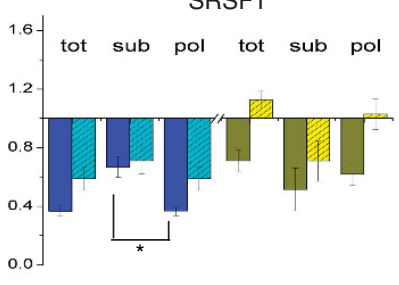

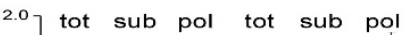

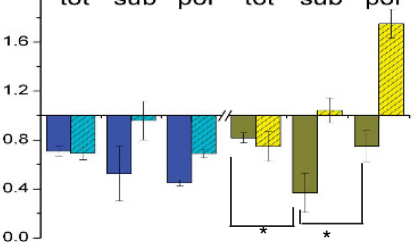

TARDBP

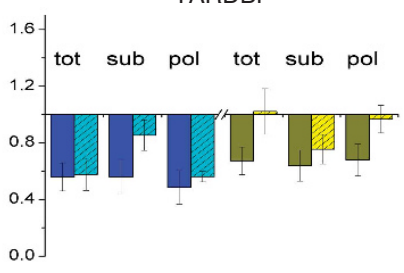

2.0 tot sub pol tot sub pol

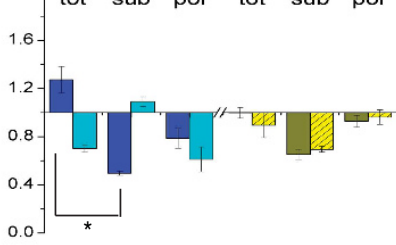

$1.6 \mathrm{E}-5$

(2)

\section{$\begin{array}{lllll}\text { MCF7vector Doxo } & \text { MCF7shp53 Doxo } & \text { MCF7vector Nutlin3 } & \text { MCF7shp53 Nutlin3A }\end{array}$}

d

$8 \mathrm{~h}$

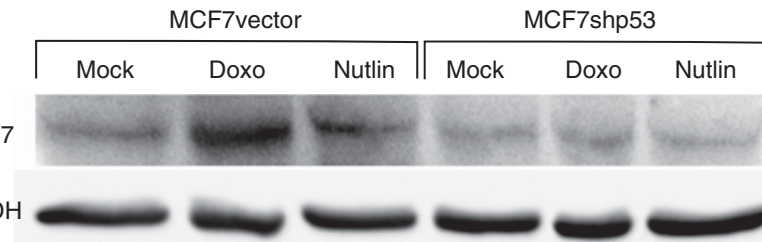

GAPDH

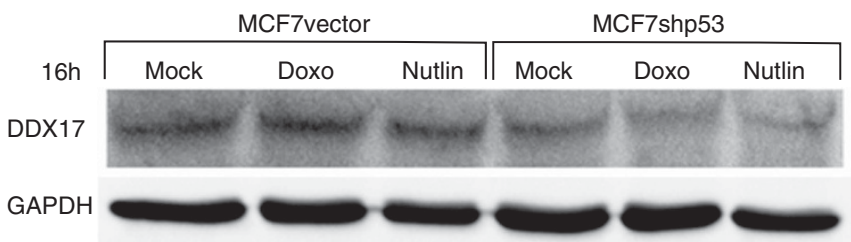

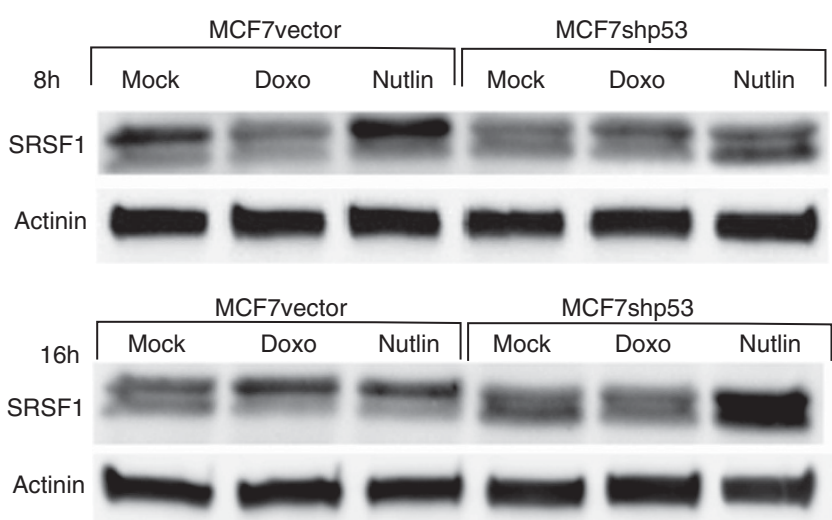

Figure 3 Subpolysomal downregulated DEGs are involved in mitosis and RNA splicing regulation. (a) Top: Venn diagrams from Figure 1b, highlighting the uncoupled DEGs that were downregulated in the subpolysomal fraction without significant changes at polysomal levels. Bottom: Venn diagram showing the level of overlap between DEGs downregulated in the subpolysomal fraction after doxorubicin and nutlin-3a treatments. (b) Gene ontology (GO) analysis on DEGs downregulated in the subpolysomal fraction that did not change in polysomal RNA. Graphs represent the most enriched GO terms obtained by DAVID analysis. $P$-values of the enriched categories are indicated. The analysis was performed on downregulated DEGs after both Doxo and Nutlin treatments. (c) DDX17, SRSF1, TARDBP and XRCC2 are indirect p53 targets. MCF7vector and MCF7shp53 cell lines were treated with Doxo and nutlin-3a for $8 \mathrm{~h}$ (upper plots) and $16 \mathrm{~h}$ (lower plots). mRNA levels were measured by qPCR in total RNA (tot), subpolysomal (sub) and polysomal (pol) fraction. Data are plotted relative to each mock condition and three reference genes. $n=3$. Means \pm S.D. are shown. ${ }^{*} P<0.05$. (d) Western Blot analysis of DDX17 (left side) and SRSF1 (right side) protein levels in MCF7vector and shp53 cells after $8 \mathrm{~h}$ (top) and $16 \mathrm{~h}$ (bottom) of treatment with Doxo or Nutlin. GAPDH and actinin were used as reference proteins for loading control. The actinin-loading control for SRSF1 is the same used as the loading control for PHPT1 in Figure 2c 
Therefore, an additional effect between p53-dependent miR-34a overexpression and p53-related reduction in the mTOR activity on YBX1 can be hypothesized.
Transcriptional and translational cross-talk between p53, YBX1, SRSF1 and c-MYC. We established that p53 indirectly modulates the expression of at least four RBPs a
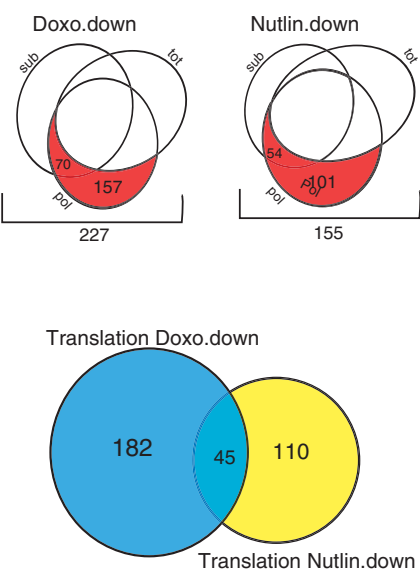

d

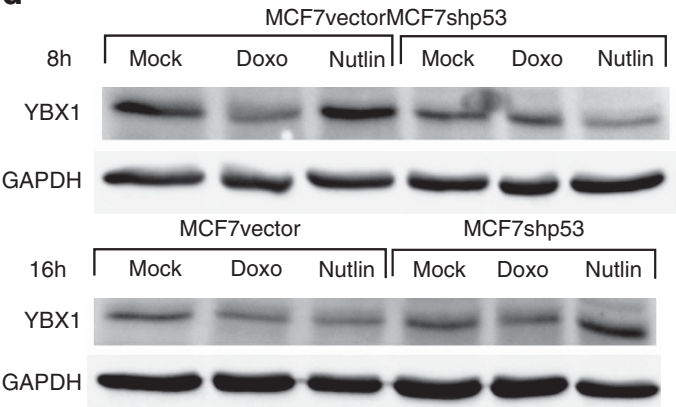

MCF7vectorMCF7shp53
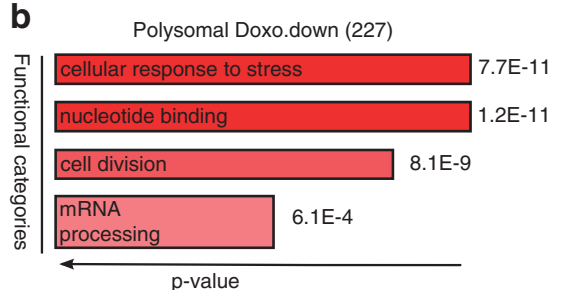

Polysomal Nutlin.down (155)

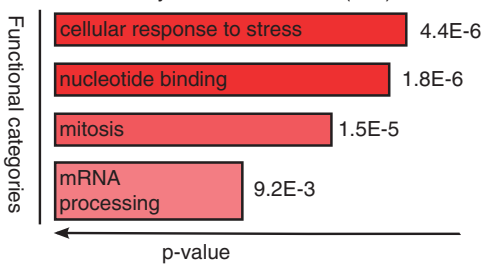

e
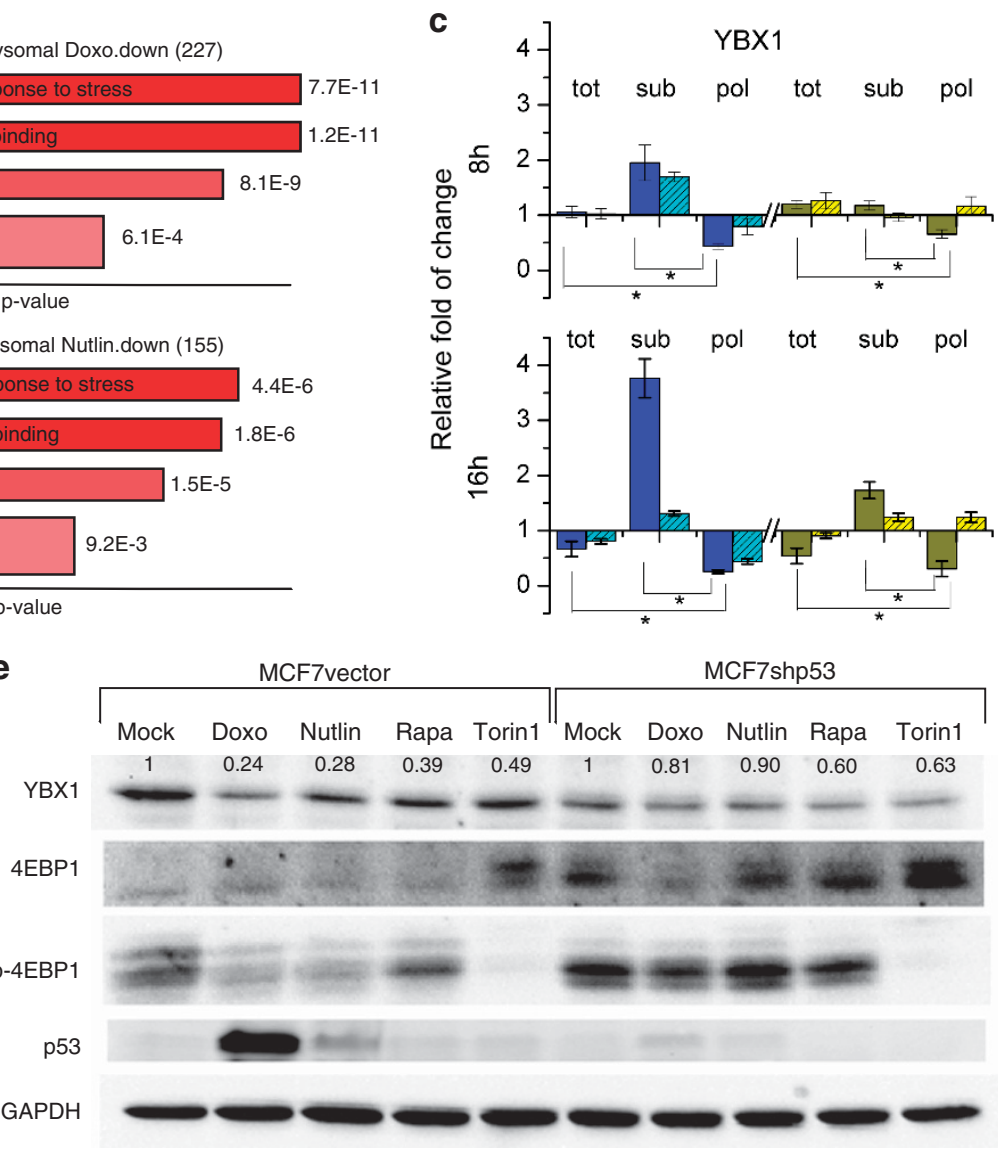

f

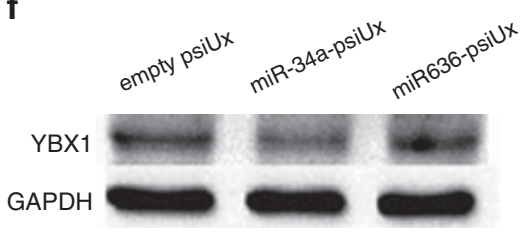

g

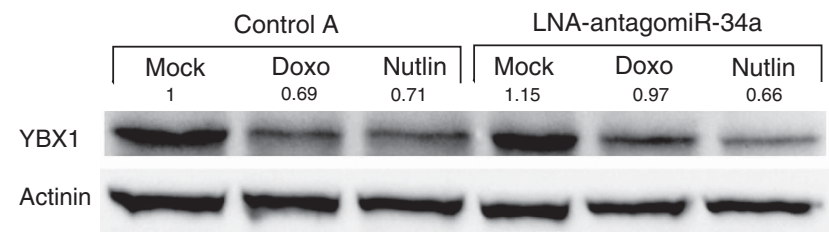

h

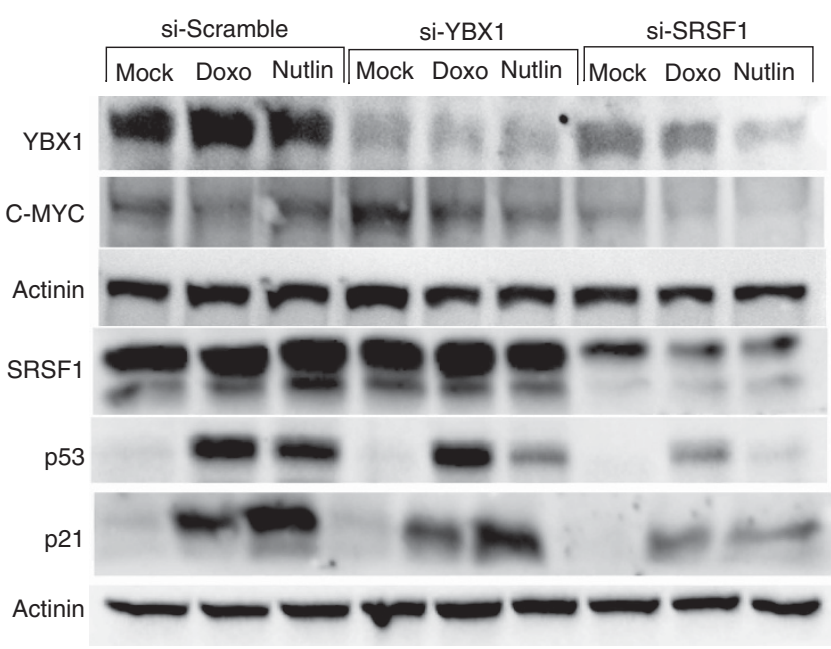


(DDX17, SRSF1, TARDBP and YBX1). By binding to their target mRNAs, RBPs could in turn contribute to the tuning of the p53-induced responses both at the transcriptional and translational levels. We chose SRSF1 and YBX1 to explore these potential regulatory modules by an siRNA approach, as they both control cell proliferation, cell-cycle progression and apoptosis ${ }^{36,37}$ (Figure 4h). We confirmed data indicating that SRSF1 reduction leads to a lower stabilization of p53 protein and to lower induction of p21. ${ }^{30,38}$ We then examined $\mathrm{C}-\mathrm{MYC}$ protein levels, given that $\mathrm{C}-\mathrm{MYC}$ translation is reported to be upregulated by $\mathrm{YBX} 1,{ }^{39}$ and SFSRF1 depletion was associated with reduced C-MYC oncogenicity. ${ }^{36}$ Interestingly, silencing SRSF1 led to a concomitant decrease in YBX1 protein and even more so in the $\mathrm{c}-\mathrm{MYC}$ protein. On the contrary, YBX1 silencing did not impact on SRSF1 or c-MYC protein levels in the mock condition.

CPEB4 and hnRNPD, mediators of translational control, are new p53 transcriptional targets. General RNA sequence features of the $5^{\prime}$ and $3^{\prime}$ UTR as well as coding sequence (CDS) influence post-transcriptional regulation of each mRNA. ${ }^{40,41}$ In order to identify potential posttranscriptional regulatory sequences embedded in the transcripts of our DEGs, we performed a distribution analysis of the length and the GC content of their $5^{\prime}$ UTR, CDS and $3^{\prime} U T R$ regions (Figure 5a). When compared with the background distribution of the whole set of human genes, translatome upregulated DEGs showed significantly shorter CDS regions and higher GC content, both in the CDS and the UTRs. On the contrary, coupled downregulated genes showed a decreased GC content, more significantly in the $3^{\prime} U T R$ region. UTR sequences of our DEGs were analyzed for the enrichment of specific regulatory elements using experimental annotation contained in the Atlas of regulatory UTR activity 2 (AURA 2) ${ }^{42}$ (Figure 5b); (Supplementary Figure S5). Target mRNAs of hnRNP-A1, $-\mathrm{C}$ and $-\mathrm{F}$ were enriched among downregulated DEGs. Conversely, hnRNPD (AUF1)-binding sites were enriched among upregulated uncoupled DEGs for both Doxo and Nutlin treatments $(\mathrm{BH}$ $P$-value: 0.00027). Furthermore, our array data identified
hnRNPD to be a downregulated coupled DEG upon Doxo treatment, a result confirmed by qPCR (Figure $5 \mathrm{c}$ ). Hence, hnRNPD should be included in the growing list of p53 target genes coding for RBPs, also considering ChIP-seq data. ${ }^{43}$

ZNF469, ZNF488 and CPEB4 were instead upregulatedcoupled RBP DEGs common to both treatments and CPEB4 was validated by $\mathrm{qPCR}$. As already reported by ChIP-seq data, ${ }^{28,43}$ we confirm that CEBP4 is a direct p53 target gene.

Other groups of transcriptionally/translationally uncoupled genes are described in Supplementary Figure S6.

\section{Discussion}

Genome-scale transcriptome analyses have been instrumental in describing the p53 gene response networks under a variety of stress responses. ${ }^{28}$ Nevertheless, the mechanism defining which cellular response is adopted remains poorly characterized. ${ }^{25}$

Here, we describe post-transcriptional gene expression control as an additional dimension to potentially shape the p53-directed gene response. Moreover, the global implications of several RBPs on that mechanism are also taken into account, given their involvement in mRNA translation. Quantitative proteomics would theoretically be an ideal tool to assess the p53-dependent translational output. Nevertheless, the coverage of proteomic studies is still a limiting factor. ${ }^{44}$ In our approach the translatome can be considered as a proxy for the proteome, although the experimental methods maintain the sensitivity typical of RNA expression studies.

To shape the downstream response networks p53 modulates RBPs that act as molecular sieves. Guided by the comparison of transcriptome and translatome data, but also considering DEGs within the free cytoplasmic pool (sub), we identified a number of RBPs that could be regulated by either Doxo (67 RBPs) or nutlin-3a (30 RBPs) or would be common to both treatments (22) (Supplementary Table S4). These 22RBPs are primary candidates for p53-directed control. Indeed, in the validation experiments

\footnotetext{
Figure 4 DEGs inhibited in translation are involved in mRNA processing and help shaping the p53-dependent response. (a) Top: Venn diagrams from Figure 1b, highlighting the uncoupled DEGs that were downregulated in the polysomal fraction without significant changes in total mRNA. Bottom: Venn diagram showing the overlap between DEGs downregulated in the polysomal fraction after Doxo and Nutlin treatments. (b) Gene ontology (GO) analysis on DEGs downregulated in the polysomal fraction that did not change in total RNA. Graphs represent the most enriched GO terms obtained by DAVID analysis. $P$-values of the enriched categories are reported. The analysis was performed on downregulated DEGs after both doxorubicin and nutlin-3a treatments. (c) YBX1 is an indirect p53 target. MCF7vector and MCF7shp53 cell lines were treated with Doxo and Nutlin for $8 \mathrm{~h}$ (upper plot) or $16 \mathrm{~h}$ (lower plot). YBX1 levels were measured by qPCR in total RNA (tot), subpolysomal (sub) and polysomal (pol) fractions. Data are plotted relative to each mock condition and three reference genes. $n=3$. Means \pm S.D. are shown. ${ }^{*} P<0.05$. (d) Western Blot analysis of YBX1 protein level in MCF7vector and shp53 cells after $8 \mathrm{~h}$ (upper panels) and $16 \mathrm{~h}$ (lower panels) of treatment with Doxo or Nutlin. GAPDH was used as reference protein for loading control, and is the same used as loading control for DDX17 in Figure 3d. (e) Western Blot analysis of MCF7vector and MCF7shp53 cell extracts after treatment with Doxo, Nutlin, rapamycin (Rapa) and Torin1. Protein levels of YBX1, total 4EBP1 and phosho(p-) 4EBP1 were measured as mTOR inhibition would lead to a reduction of 4EBP1 phosphorylation (p-4EBP1) and the consequent inhibition of cap-dependent translation. As control, p53 protein levels were measured. p53 is stabilized after $16 \mathrm{~h}$ of treatment with Doxo and Nutlin, whereas the treatment with mTOR inhibitors does not have an impact on p53 protein levels. GAPDH was used as a reference protein for loading control. For YBX1, numbers above the immunoreactive bands represent the relative amount of proteins normalized against both the reference protein and the mock condition (set to 1 separately for each cell line). (f) Ectopic overexpression of miR-34a in MCF7vector cell line. The empty miR-expression plasmid psiUx condition was used as negative control. miR-636 was overexpressed as an additional control of miR-34a specificity. GAPDH was used as a reference protein for loading control. (g) YBX1 protein levels in MCF7vector cells transfected with the negative Control A or an LNA-antagomiR-34a and treated after $48 \mathrm{~h}$ with Doxo or Nutlin. Actinin was used as a reference protein for loading control. Numbers above the immunoreactive bands represent the relative amount of proteins normalized using both the reference protein and the mock condition in the control experiment (set to 1). (h) Western Blot analysis on MCF7vector cell extracts after silencing YBX1, SRSF1 or scramble control, either in the mock condition or after Doxo or Nutlin treatment. Protein levels of YBX1 and SRSF1 confirm the silencing. c-MYC protein levels were measured because it is a recognized downstream target of both YBX1 and SRSF1. As additional controls, p53 as well as p21 levels were measured. Actinin was used as a reference protein for loading control
} 
a
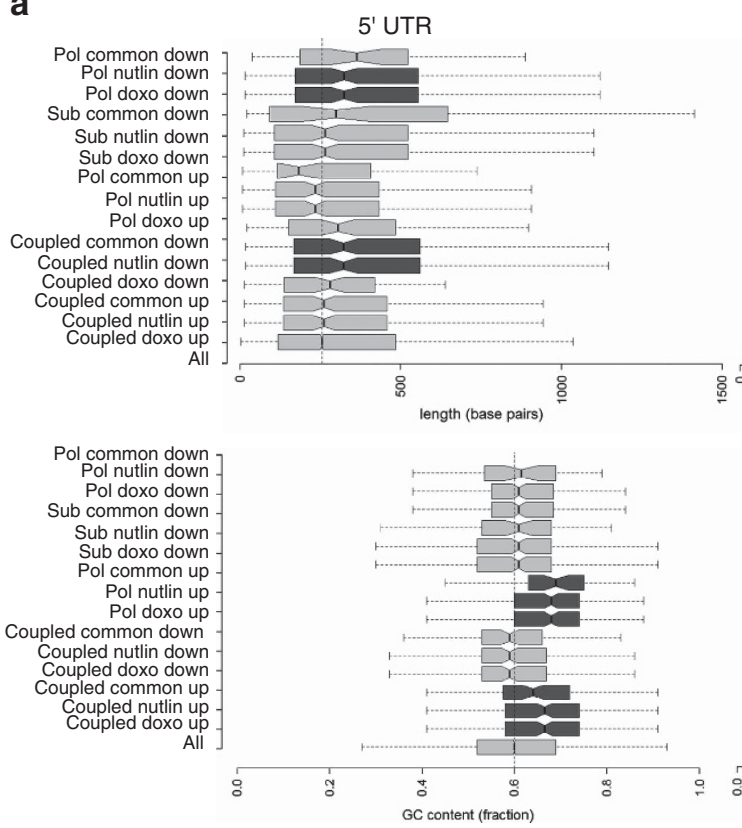

b

Enrichment of post-transcriptional regulators

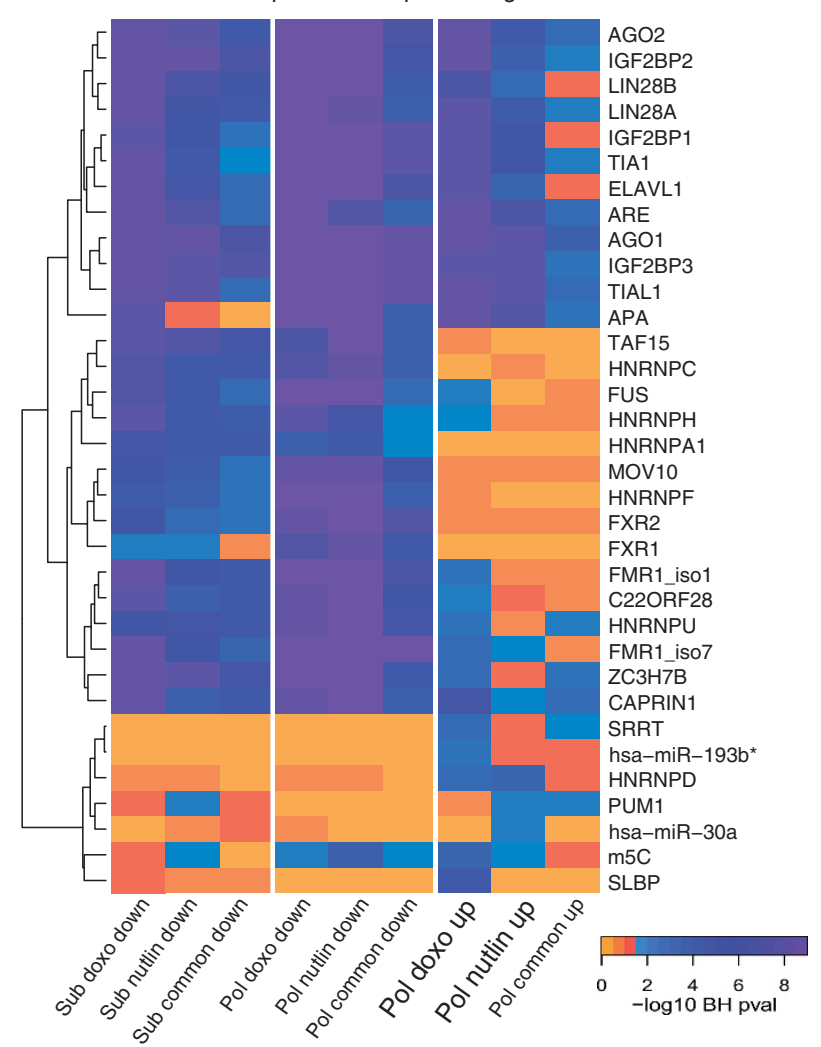

CDS
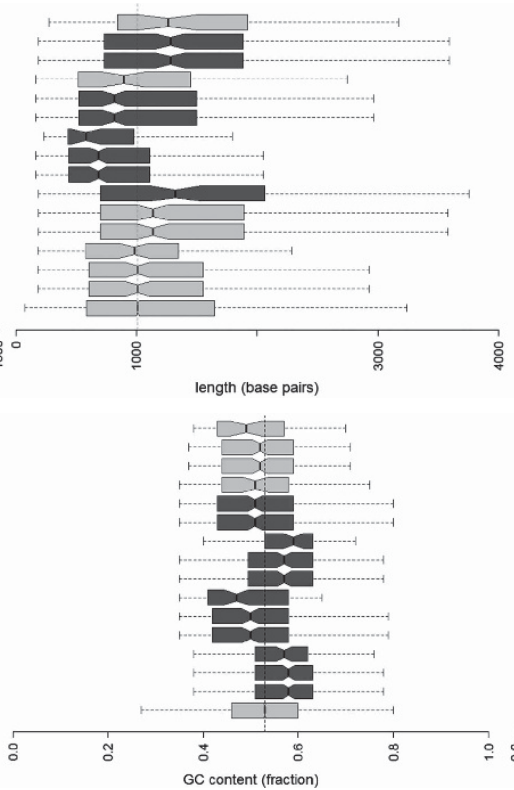

C

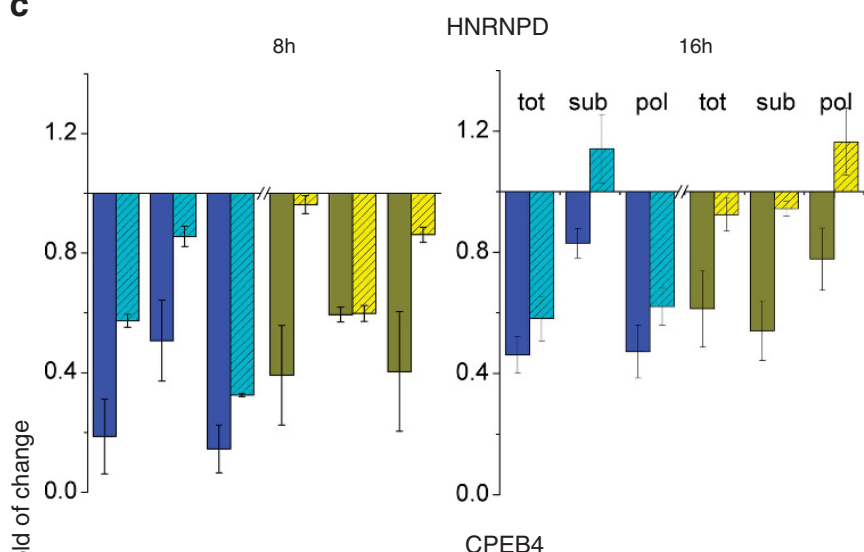

$8 \mathrm{~h}$

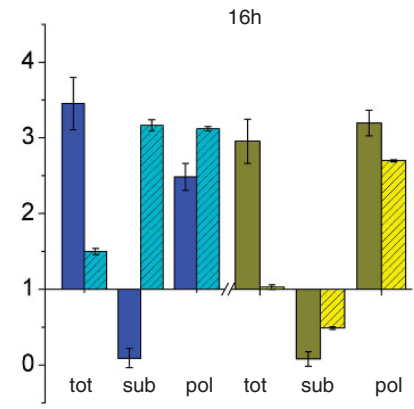

Figure 5 Enrichment of sequence features or of regulatory elements in the UTRs or coding sequence of uncoupled DEGs. (a) The upper plots show the length distribution for the $5^{\prime}$-untranslated region (UTR), coding region (CDS) and $3^{\prime}$-UTR of the indicated list of our DEGs. Common refers to the overlap between Doxo and Nutlin DEGs. The lower plots present the GC content for each of these categories. All distributions are compared with the background distribution of the whole set of human genes (all). The significant shifts in distribution are shown in dark gray (Mann-Whitney test, $P$-value $<0.01$ ). (b) Heatmap based on the enrichment $P$-values adjusted for multiple testing with the Benjamini-Hochberg method. DEGs upregulated in the subpolysomal fraction, upregulated in the polysomal fraction or downregulated in the polysomal fraction are presented for each treatment (Doxo and Nutlin). The enrichment of regulatory elements for DEGs common to both treatments are also presented, referred to as 'common'. (c) hnRNPD and CPEB4 are direct p53 targets. MCF7vector and MCF7shp53 cell lines were treated with Doxo and Nutlin for 8 or $16 \mathrm{~h}$. hnRNPD and CPEB4 levels were measured by qPCR in total RNA (tot), subpolysomal (sub) and polysomal (pol) fractions. Data are plotted relative to each mock condition and three reference genes. $n=3$. Means \pm S.D. are shown 
we confirmed YBX1, SRSF1, DDX17, TARDBP, HNRNPD and CEBP4 as targets of p53-dependent modulation at the total mRNA or polysomal mRNA levels or both (Figure 6). In particular, we focused on targets that could directly or indirectly modulate p53 functions, either by acting on the p53 mRNA or on the mRNAs of p53 target genes.

Interestingly, hnRNPD had already been reported to target and destabilize the mRNAs of $\mathrm{p} 53,{ }^{45} \mathrm{BAX}$ and other important cancer genes, often in a reciprocal, alternating association with HuR, an mRNA-stabilizing factor. ${ }^{45}$ We propose that through the transcriptional downregulation of hnRNPD, p53 can engage a positive feedback and potentially also a feed-forward regulatory loop. Consistently, we found enrichment for hnRNPD target mRNAs among the group of translationally upregulated DEGs (Figure 5a).

CPEB4 was an upregulated-coupled DEG whose enhanced expression was abated by p53 silencing. Notably, CPEB4 is a member of the CPEB family, and CPEB1, functionally related with CPEB4, was shown to sustain p53 translation, thereby participating in the activation of the senescence response. ${ }^{46,47}$ We suggest that p53 could impact its own translation fitness and functions via its direct target gene CPEB4.

DDX17, TARDBP, SRSF1 and YBX1 are confirmed as modulated at the post-transcriptional level. Overall, in almost all the analyses, protein levels reflect the subpolysomal or polysomal mRNA changes, suggesting that these mRNA variations could have a significant impact on the final proteome. Although TARDBP functions are still under investigation, DDX17/p72 is a putative RNA helicase ${ }^{48}$ that by interacting with DDX5 (p68) can act as a modulator of p53-dependent transcription and DNA damage response.
SRSF1 was repressed by both doxorubicin and nutlin-3a treatments particularly in the subpolysomal RNA fraction, and the p53-dependent negative modulation was apparent comparing MCF7vector with MCF7shp53 cells. As it was recently reported that SRSF1 overexpression provides resistance to oncogenic transformation via stabilization of $\mathrm{p} 53,{ }^{30,49}$ we propose that we have uncovered a negative feedback loop by which p53 inhibits a positive regulator.

We explored in more detail two mechanisms linking p53 activation with YBX1 mRNA and protein downregulation, namely the inhibition of the mTOR pathway ${ }^{32}$ and the upregulation of miR-34a. $^{12}$ Although p53 can negatively impact on mTOR, via the transcriptional activation of SESN1 and SESN2, ${ }^{33}$ the high dependency of mTOR function on the cell metabolic state can also influence our results. The dynamics by which p53 modulates all these RBPs is an additional, critical point. Here, we measured the expression of these genes also after $8 \mathrm{~h}$ of treatments to begin exploring this issue. Temporal differences in p53 stabilization upon doxorubicin and nutlin-3a treatments have been already reported $^{50}$ and are confirmed by our analysis. Overall, the qPCR results revealed correlations (e.g., p21, PUMA, TRIAP1, TRAF4, XRCC2, hnRNPD and CPEBP4) as well as differences (MDM2, PHPT1, TP53RK, GADD45G, DDX17, SRSF1, TARDBP and YBX1), between the two time points and also between mRNA and protein levels.

Further investigations are needed to clarify the specific impact of RBPs on post-transcriptional regulation after p53 activation. In our experiments SRSF1 silencing led to a decrease also in YBX1 protein levels, suggesting a cross-talk between the two RBPs via unexplored mechanisms, and this resulted in down-modulation of c-MYC. Hence, p53dependent negative regulation of both YBX1 and SRSF1,

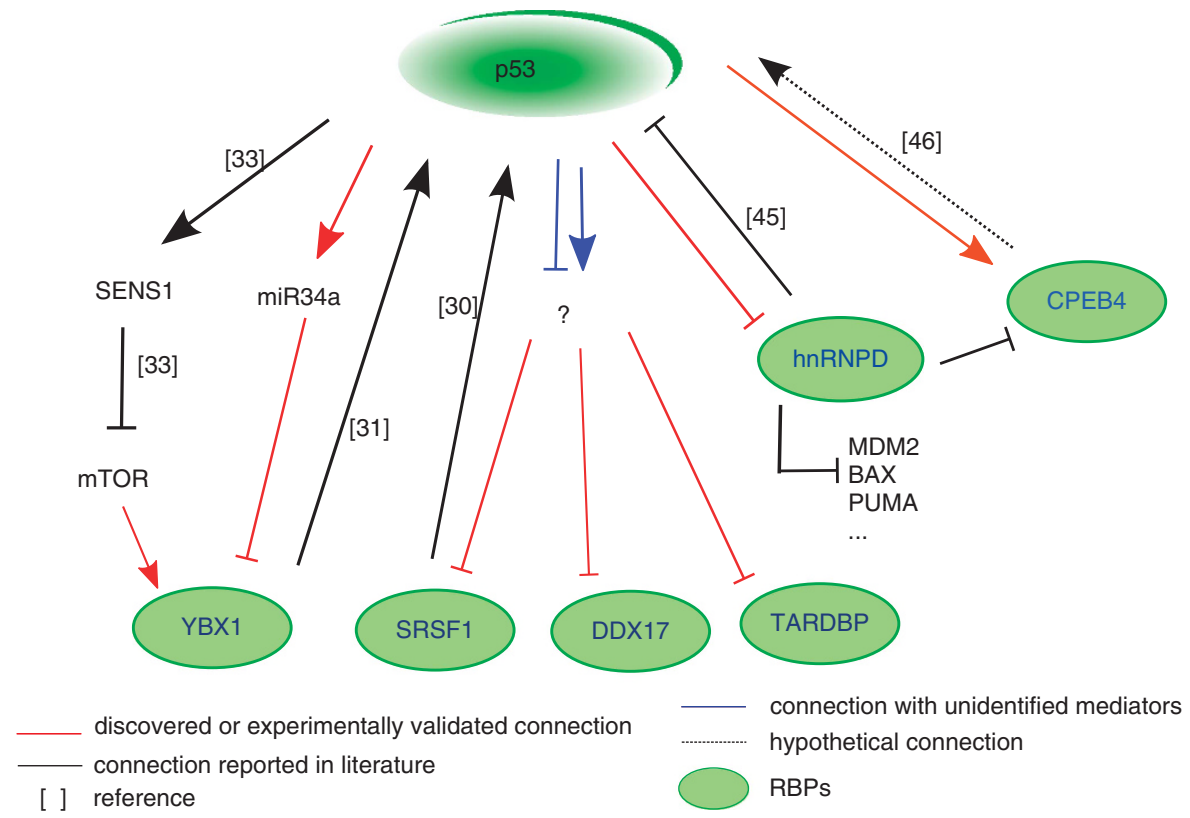

Figure 6 Five RBPs are regulated by p53 and can contribute to post-transcriptional control of p53 responses. Cartoon highlighting functional links between wild-type p53 and six RBPs that are established or confirmed in our work. Solid red lines and arrows represent functional interactions discovered or experimentally validated in this work. Black lines represent functional interactions reported in the literature (citation numbers are near those arrows). Blue lines are used when the mediators of the functional interactions are unknown. The impact of CPEB4 on p53 is hypothetical, based on results with the related CPEB1 protein 
could lead to repression of c-MYC, and contribute to cell cycle arrest. Importantly, for C-MYC and potentially many other targets, p53 could also impact indirectly on mRNA translation efficiency via transcriptional inhibition of the Fibrillarin gene. ${ }^{51}$

\section{Apoptosis can be regulated also at the level of transla- tion efficiency of p53 target genes. Gene ontology of DEGs that were uncoupled and upregulated only in the} polysomal fraction revealed an enrichment for the term 'apoptosis', which was even more significant in cells treated with nutlin-3a. This finding uncovers a new layer of complexity in the modulation of the classical p53-dependent apoptosis (Supplementary Figure S7). Several studies have shown that transcriptional activation of apoptosis gene targets can be influenced by selective cofactors or specific post-translational modifications of the p53 protein. ${ }^{24} \mathrm{We}$ suggest that translational controls may promote the synthesis of those pro-apoptotic proteins contributing to the actual induction of programmed cell death. Recently, Ribo-seq was used to profile MCF7 cells treated with nutlin-3a. ${ }^{52}$ Ribo-seq maps ribosome-protected fragments, but, unlike polysomal profiling, it does not separate actively translating polysomes from monosomes (80S), nor does it address subpolysomal RNA. Consistently with our results, downregulation of cell-cycle genes was observed but the modulation of apoptosis or mRNA-processing pathways was not apparent in Ribo-seq data.

In summary, our analysis of uncoupled mRNAs, namely mRNAs undergoing translational control, reveals a large number of new indirect p53-regulated targets that would not have been identified through a traditional transcriptome study. On the basis of the functions of these genes, it becomes apparent that selectivity at the level of mRNA translation, in addition to transcriptional selectivity, is a critical contributing factor in the shaping of p53-directed responses and at least six RBPs directly or indirectly modulated by p53 may be implicated. Our study opens up a scenario where further investigations will clarify the impact of p53-dependent post-transcriptional regulation as well as the involvement of RBPs on cellular outcomes.

\section{Materials and Methods \\ Cell lines and culture conditions. MCF7 cells stably expressing an shRNA targeting p53 (MCF7shp53) or control cells (MCF7vector) were kindly provided by Dr Agami. ${ }^{20}$ Cells were normally maintained in RPMI (Gibco, Life Technologies, Milan, Italy) supplemented with $10 \%$ FBS, antibiotics (100 units/ml penicillin plus $100 \mathrm{mg} / \mathrm{ml}$ streptomycin) and $2 \mathrm{mM} \mathrm{L-glutamine.} \mathrm{Puromycin} \mathrm{(Sigma-}$ Aldrich, Milan, Italy) was used to maintain the selection, at $0.5 \mu \mathrm{g} / \mathrm{ml}$ as final concentration.}

Polysomal RNA fractionation and extraction. MCF7vector cells $\left(3.5 \times 10^{6}\right)$ were seeded into $10 \mathrm{~cm}$ tissue culture dishes and allowed to reach $70-80 \%$ confluence before treatment with $1.5 \mu \mathrm{M}$ doxorubicin (Doxo) or $10 \mu \mathrm{M}$ nutlin-3a (Nutlin). Doxo was purchased from Sigma-Aldrich, whereas nutlin-3a was obtained from Alexis Biochemicals (Enzo Life Science, Exeter, UK). After 8 or $16 \mathrm{~h}$, polysomal separation was performed as previously described. ${ }^{53}$ Briefly, samples were loaded in 15-50\% linear sucrose gradients, ultra-centrifuged and fractionated with an automated fraction collector. All the fractions containing subpolysomal or polysomal RNA were identified and pooled in two separate tubes. RNA was purified by extraction with 1 volume of phenol-chloroform and adding a washing step in $70 \% \mathrm{v} / \mathrm{v}$ ethanol in order to remove phenol contaminations. DNAse treatment (RNase-Free DNase Set, Qiagen, Hilden, Germany) was performed to remove DNA contamination after the RNA extraction. Three biological replicates were performed. For validation studies, all these steps were repeated also for the MCF7shp53 cell line, seeding $2 \times 10^{6}$ cells/dish.

Total RNA extraction. MCF7vector and MCF7shp53 cell lines were seeded into six-well plates and allowed to reach $70-80 \%$ of confluency before treating with $1.5 \mu \mathrm{M}$ Doxo or $10 \mu \mathrm{M}$ Nutlin. After 8 or $16 \mathrm{~h}$ of treatment, cells were harvested and total RNA was extracted using the Agilent Total RNA Isolation Mini Kit (Agilent Technologies, Milan, Italy) according to the manufacturer's instructions. In-column DNAse treatment (RNase-Free DNase Set, Qiagen) was performed to remove DNA contamination during the extraction. Three biological samples were analyzed.

Microarray hybridization and data analysis. Purity of all extracted RNAs (A260/A280 value of 1.8-2.1) and concentrations were measured using the Nanodrop spectrophotometer. An additional quality control was performed with the Agilent 2100 Bioanalyzer (Agilent Technologies) discarding RNA preparations with RIN (RNA integrity number) value $<8$. mRNAs extracted after $16 \mathrm{~h}$ of treatment with Doxo or Nutlin were hybridized to an Agilent-014850 Whole Human Genome Microarray 4x44K G4112F-Probe following the manufacturer's protocol. Raw data and procedures were deposited in Gene Expression Omnibus (GSE50650). That output was analyzed with the tRanslatome package ${ }^{54}$ using the Limma method (http://www.bepress.com/sagmb/vol3/iss1/art3) comparing each treatment of every tested RNAs with the mock condition. Moreover, Supplementary Table S5 shows the detailed list of all our DEGs. For all further analysis on DEGs, two thresholds were set for each comparison: (1) $\log _{2}$ (fold change) $>1$ and $<-1$ for upregulated and downregulated genes, respectively; (2) Benjamini-Hochberg (BH) corrected $P$-value $<0.05$.

Gene ontology and pathway analysis of DEGs. Gene-annotation enrichment analysis for all our selected categories of coupled or uncoupled DEGs was performed with the DAVID resource. ${ }^{55}$ The significance of overrepresentation was determined at a false discovery rate of $5 \%$ with $\mathrm{BH}$ multiple testing correction and an enrichment score $>1.5$. All pathways analyses were performed using ingenuity pathway analysis (www.ingenuity.com). Only direct interactions were considered in setting parameters.

Analysis of UTR sequence features. UTR and CDS sequences were downloaded from the UCSC Genome Browser (http://genome.ucsc.edu/), assembly GRC37/hg19. For each HGNC gene the longest transcript variant was selected as representative of the gene. Distribution analysis was performed on the length and GC content of $5^{\prime} U T R, C D S$ and $3^{\prime} U T R$ regions of the lists of DEGs. All the distributions were compared with the background distribution corresponding to the whole set of human genes, and significant shifts were identified with the Mann-Whitney test, selecting a 0.01 significance threshold on the resulting $P$-value.

Analysis of $5^{\prime}-3^{\prime}$ UTRs and of RBP genes. The AURA 2 database (http://aura.science.unitn.it/) was used to perform the analysis of the enrichment of regulatory elements at the $5^{\prime}-3^{\prime} U T R$ of coupled or uncoupled DEGs. Given that AURA 2 is a database containing only experimentally validated post-transcriptional interactions at the UTR level, we used AURA to select enriched RBPs for further validations. The presented enrichment $P$-values were adjusted for multiple testing with the BH-method. We matched our DEG classes with a restricted RBPs' list to obtain the number of RBP genes that were modulated after Doxo and nutlin-3a treatments. The restricted list was compiled including all canonical RBPs (i.e., proteins containing at least one recognized RBP motif), translation factors and non-canonical RBPs reported in previous studies. ${ }^{56,57}$

RT-qPCR reaction. cDNA was generated from $1 \mu \mathrm{g}$ of RNA using the RevertAid First Strand cDNA Synthesis Kit (Fermentas, Milan, Italy) in $20 \mu$ l final volume following manufacturer's instructions. All qPCR assays were performed on a CFX Touch Real-Time PCR Detection System (Bio-Rad, Milan, Italy) in a 384-well plate format. Assays contained 2X KAPA Probe FAST qPCR Master Mix (Kapa Biosystems, Resnova, Rome, Italy), $20 \times$ PrimeTime ZEN DoubleQuenched Probes Assay (IDT, Tema Ricerca, Bologna, Italy) and $25 \mathrm{ng}$ of cDNA. Primers are all commercially available according to their catalog number. In addition, we validated some targets using the $2 \times$ KAPA SYBR FAST qPCR Kit (Kapa Biosystems, Resnova) and specific primers purchased from Eurofins (MWG, Operon, Ebersberg, Germany). The list of primers is presented in 
Supplementary Table S6. All these primers were validated according to the MIQE guidelines. ${ }^{58}$ We present the mRNA quantification relative to the mock condition for each fraction (tot, sub and pol) in order to highlight changes upon treatment. To clarify variation in the mock variation, the $\Delta \mathrm{Cq}$ data of the mock condition are reported in Supplementary Table S7. The relative quantification was obtained using the comparative $\mathrm{Cq}$ method $(\Delta \Delta \mathrm{Cq})$, where glyceraldehyde 3-phosphate dehydrogenase (GAPDH), $\beta$-2microglobulin and tyrosine 3-Monooxygenase/ TRYPTOPHAN 5-Monooxygenase Activation Protein, Zeta Polypeptide (YWHAZ) served as reference genes. The relative folds of change were analyzed using a $t$-test approach considering three biological replicates $(P<0.05)$.

Antibodies and western blot analysis. Antibodies used for western blot analysis were p53 (DO-1), p21(C-19), YBX1(59-Q), 4EBP1(R-113), GAPDH(6C5), alpha-Actinin (B-19), SRSF1 (3G268), MYC (9E10) and PHPT1 (N-23) from Santa Cruz Biotechnology (Heidelberg, Germany), p-4EBP1(Thr37/46) from Cell Signaling Technology (Milan, Italy) and DDX17 (ab70184) from Abcam (Cambridge, UK). MCF7vector and MCF7shp53 cells were seeded into six-well plates and allowed to reach $70-80 \%$ of confluency before treating with Doxo $(1.5 \mu \mathrm{M})$, Nutlin $(10 \mu \mathrm{M})$ for 16 or $8 \mathrm{~h}$ and rapamycin $(250 \mathrm{nM})$ and Torin $1(250 \mathrm{nM})$ for $2 \mathrm{~h}$. The concentration and time point used for rapamycin and Torin1 are based on a previous paper. ${ }^{59}$ Rapamycin (Sigma-Aldrich)-Torin1 (Tocris Bioscience, Bristol, UK). Proteins were extracted using RIPA buffer as previously described, ${ }^{2}$ supplemented with protease inhibitors and phosphatase inhibitor cocktail2 (Sigma-Aldrich) and quantified using the BCA assay (Thermo Scientific, Pierce, Milan, Italy). The relative molecular mass of the immunoreactive bands was determined using PageRuler Plus Prestained Protein Ladder (Fermentas). The semi-quantitative analysis was performed using GAPDH or Actinin as reference proteins for loading control.

Silencing of YBX1 and SRSF1 proteins. To perform YBX1 or SRSF1 silencing, we used DsiRNA Duplex purchased from IDT (si-YBX1: HSC.RNAI.N004559.12.3, si-SRSF1: HSC.RNAI.N006924.12.1). MCF7vector cells were seeded into six-well plates and allowed to reach $30-40 \%$ of confluence. After $24 \mathrm{~h}, 25 \mathrm{nM}$ of the different DsiRNAs were transfected using INTERFERin (Polyplus, Euroclone, Milan, Italy). As a negative control, we transfected cells with the si-scramble si.NC1 at the same final concentration. Fifty-six hours after the transfection, cells were treated with doxorubicin and nutlin. Antisense effects were assessed $16 \mathrm{~h}$ after the treatments, thats is, $72 \mathrm{~h}$ after transfection.

Apoptosis assays. MCF7vector cells were seeded in 96-well plates (Corning, Lowell, MA, USA) at the density of 15000 cells/well. After $24 \mathrm{~h}$, cells were treated with $0.75,1.5$ and $3 \mu \mathrm{M}$ of Doxo and $5 \mu \mathrm{M}, 10 \mu \mathrm{M}$ and $15 \mu \mathrm{M}$ of Nutlin. After $16 \mathrm{~h}$, cells were exposed to $10 \mu /$ well of Cell Proliferation Reagent WST-1 (Roche, Milan, Italy) for $30 \mathrm{~min}$ before measuring the absorbance at 460 and $600 \mathrm{~nm}$ using an Infinite 200 PRO microplate reader (TECAN, Milan, Italy). A reduction in the absorbance signal is proportional to a reduction in the activity of mitochondrial dehydrogenases that is considered as a marker of cell viabilty. Doxo $(1.5 \mu \mathrm{M})$ and $10 \mu \mathrm{M}$ Nutlin were chosen for all subsequent experiments. We chose a $16 \mathrm{~h}$ time point after doxorubicin and nutlin treatment of MCF7 cells at relatively low doses because we were interested in identifying p53-directed or stressresponse-directed mechanisms of post-transcriptional control. For Fluorescenceactivated cell sorting analysis, MCF7vector cells were seeded into 10-cm dishes and treated the following day. In order to have more information about cells viability, we recovered and analyzed also cells that were in suspension after the treatments. The FITC AnnexinV Apoptosis Detection kit I (BD Pharmingen, Milan, Italy) was used for the staining following the manufacturer's protocol. TO-PRO-3 lodide $(1 \mu \mathrm{M})(642 / 661)$ was used as a nucleic acid dye (Life Technologies).

miRNA extraction and quantification. MCF7vector and MCF7shp53 cells were seeded into six-well plates and allowed to reach $70-80 \%$ confluence before treating with Doxo or Nutlin. After $16 \mathrm{~h}$, cells were harvested and total RNA was extracted using $300 \mu \mathrm{l}$ of TRIZOL reagent (Invitrogen, Life Technologies). After $5 \mathrm{~min}$ of incubation at room temperature, $60 \mu \mathrm{l}$ of chloroform were added, followed by another incubation step of $3 \mathrm{~min}$ at room temperature. Three different phases were obtained following centrifugation at $4^{\circ} \mathrm{C}$ for $15 \mathrm{~min}$ at $12000 \times \mathrm{g}$. We recovered only the aqueous phase containing the RNA to continue with isopropanol precipitation and subsequent ethanol $75 \%$ wash. mRNA quality was controlled as described above. Mature miR expression levels were quantified using pre-made Exiqon assays, using the small nuclear snRNA U6 as reference and following the manufacturer's instructions for CDNA reaction (Universal cDNA Synthesis kit, Exiqon, Woburn, MA, USA) and qPCR with the ExiLENT SYBRGreen Master Mix (Exiqon).

miRNA overexpression. To overexpress pre-miR-34a, we used an siRNA-expressing vector (psiUx) based on the strong and ubiquitous RNA Pollldependent promoter of the human U1 small nuclear RNA (snRNA) gene. ${ }^{60}$ Transfection of the empty psiUx was used as a control. ${ }^{61}$ miR-636 was overexpressed as an additional control to confirm a specific effect of miR-34a. MCF7vector cells were seeded into six-well plates. After $24 \mathrm{~h}$, cells were transfected with the different plasmids using FuGENE HD Transfection Reagent (Promega, Milan, Italy). Forty-eight hours after the transfection, cells were harvested and miRNAs or proteins were extracted for quantification assays, as described in the previous sections.

miRNA inhibition. To inhibit miR-34a, MCF7vector cell lines were seeded into six-well plates. When cells reached $30-40 \%$ of confluence, miRCURY LNA miR-34a Inhibitor (Exiqon) was transfected using INTERFERin transfection reagent (Polyplus). After optimization experiments, we used $50 \mathrm{nM}$ of miR-34a inhibitor final concentration for the transfection. As a negative control, we transfected cells with miRCURY LNA microRNA Inhibitor Negative Control A at the same final concentration. Thirty-two hours after the transfection, cells were treated with Doxo and Nutlin. Effects were assessed $16 \mathrm{~h}$ after the treatments, that is, $48 \mathrm{~h}$ after transfection.

\section{Conflict of Interest}

The authors declare no conflict of interest.

Acknowledgements. We thank Dr. Valentina Adami (HTS facility at $\mathrm{CIBIO}$ ) for technical assistance with the microarray experiments and Dr. Isabella Pesce with the FACS experiments (Cell Analysis and Separation facility at CIBIO). We thank Drs Matthew Galbraith, Mattia Lion, Daniel Menendez, Michael A Resnick and Maria del Huerto Flammia for critical evaluation of the paper content and style. We are grateful to Drs Erik Dassi and Alessandro Quattrone for access to AURA 2. This work was partially supported by the Italian Association for Cancer Research, AIRC, (IG\# 12869) and by CIBIO start-up funds.

\section{Author contributions}

SZ carried out the experiments, analyzed the microarray data and cowrote the manuscript. TT contributed to microarray data analysis. CP participated in validation experiments and draft correction. YC participated in the coordination of the study. $A B$ conceived the study, and participated in its design. Al conceived the study, participated in its coordination and cowrote the manuscript. All authors read and approved the final manuscript.

1. Levine aJ, Hu W, Feng Z. The P53 pathway: what questions remain to be explored? Cell Death Differ 2006; 13: 1027-1036.

2. Lion M, Bisio A, Tebaldi T, De Sanctis V, Menendez D, Resnick MA et al. Interaction between p53 and estradiol pathways in transcriptional responses to chemotherapeutics. Cell Cycle 2013; 12: 1211-1224.

3. Sonenberg N, Hinnebusch AG. Regulation of translation initiation in eukaryotes: mechanisms and biological targets. Cell 2009; 136: 731-745.

4. Hinnebusch AG, Lorsch JR. The mechanism of eukaryotic translation initiation: new insights and challenges. Cold Spring Harb Perspect Biol 2012; 4: a011544.

5. Dever TE, Green R. The elongation, termination, and recycling phases of translation in eukaryotes. Cold Spring Harb Perspect Biol 2012; 4: a013706.

6. Glisovic T, Bachorik JL, Yong J, Dreyfuss G. RNA-binding proteins and post-transcriptional gene regulation. FEBS Lett 2008; 582: 1977-1986.

7. He L, Hannon GJ. MicroRNAs: small RNAs with a big role in gene regulation. Nat Rev Genet 2004; 5: 522-531

8. Provenzani A, Fronza R, Loreni F, Pascale A, Amadio M, Quattrone A. Global alterations in mRNA polysomal recruitment in a cell model of colorectal cancer progression to metastasis. Carcinogenesis 2006; 27: 1323-1333.

9. Tebaldi T, Re A, Viero G, Pegoretti I, Passerini A, Blanzieri E et al. Widespread uncoupling between transcriptome and translatome variations after a stimulus in mammalian cells. BMC Genomics 2012; 13: 220

10. Le MTN, Teh C, Shyh-Chang N, Xie H, Zhou B, Korzh V et al. MicroRNA-125b is a novel negative regulator of p53. Genes Dev 2009 862-876. 
11. Chen J, Kastan MB. $5^{\prime}-3^{\prime}$-UTR interactions regulate p53 mRNA translation and provide a target for modulating p53 induction after DNA damage. Genes Dev 2010; 24: 2146-2156.

12. Freeman Ja, Espinosa JM. The impact of post-transcriptional regulation in the p53 network. Brief Funct Genomics 2013; 12: 46-57.

13. Scoumanne A, Cho SJ, Zhang J, Chen X. The cyclin-dependent kinase inhibitor p21 is regulated by RNA-binding protein PCBP4 via mRNA stability. Nucleic Acids Res 2011; 39 213-224.

14. Wang W, Furneaux $\mathrm{H}$, Cheng $\mathrm{H}$, Caldwell MC, Hutter $\mathrm{D}$, Liu $\mathrm{Y}$ et al. HuR regulates $\mathrm{p} 21$ mRNA stabilization by UV light. Mol Cell Biol 2000; 20: 760-769.

15. Ghosh M, Aguila HL, Michaud J, Ai Y, Wu MT, Hemmes A et al. Essential role of the RNA-binding protein HuR in progenitor cell survival in mice. J Clin Invest 2009; 119 3530-3543.

16. Suzuki HI, Yamagata K, Sugimoto K, Iwamoto T, Kato S, Miyazono K. Modulation of microRNA processing by p53. Nature 2009; 460: 529-533.

17. Zhang J, Cho SJ, Chen X. RNPC1, an RNA-binding protein and a target of the p53 family, regulates p63 expression through mRNA stability. Proc Natl Acad Sci USA 2010; 107: 1-6. www.pnas.org/cgi/doi/10.1073/pnas.0912594107.

18. Rahman-roblick R, Roblick UJ, Hellman U, Conrotto P, Liu T, Becker S et al. p53 targets identified by protein expression profiling. Proc Natl Acad Sci USA 2007; 104 5401-5406.

19. Arava Y. Isolation of polysomal RNA for microarray analysis. Methods Mol Biol 2003; 224: 79-87.

20. Brummelkamp TR, Bernards R, Agami R. A system for stable expression of short interfering RNAs in mammalian cells. Science 2002; 296: 550-553.

21. Melamed D, Eliyahu E, Arava Y, Bähler J. Exploring translation regulation by global analysis of ribosomal association. Methods 2009; 48: 301-305.

22. Ek P, Pettersson G, Ek B, Gong F, Li JP, Zetterqvist O. Identification and characterization of a mammalian 14-kDa phosphohistidine phosphatase. Eur J Biochem 2002; 269: 5016-5023.

23. Peterson D, Lee J, Lei XC, Forrest WF, Davis DP, Jackson PK et al. A chemosensitization screen identifies TP53RK, a kinase that restrains apoptosis after mitotic stress. Cancer Res 2010; 70: 6325-6335.

24. Riley $\mathrm{T}$, Sontag $\mathrm{E}$, Chen $\mathrm{P}$, Levine A. Transcriptional control of human p53-regulated genes. Nat Rev Mol Cell Biol 2008; 9: 402-412.

25. Andrysik Z, Kim J, Tan AC, Espinosa JM. A genetic screen identifies TCF3/E2A and TRIAP1 as pathway-specific regulators of the cellular response to p53 activation. Cell Rep 2013; 3: 1346-1354

26. Sax JK, El-Deiry WS. Identification and characterization of the cytoplasmic protein TRAF4 as a p53-regulated proapoptotic gene. J Biol Chem 2003; 278: 36435-36444.

27. Salvador JM, Brown-Clay JD, Fornace AJ. Gadd45 in stress signaling, cell cycle control, and apoptosis. Adv Exp Med Biol 2013; 793: 1-19.

28. Nikulenkov F, Spinnler C, Li H, Tonelli C, Shi Y, Turunen M et al. Insights into p53 transcriptional function via genome-wide chromatin occupancy and gene expression analysis. Cell Death Differ 2012; 19: 1992-2002.

29. Xu K, Chen Z, Qin C, Song X. miR-7 inhibits colorectal cancer cell proliferation and induces apoptosis by targeting XRCC2. Onco Targets Ther 2014; 7: 325-332.

30. Fregoso OI, Das S, Akerman M, Krainer AR. Splicing-factor oncoprotein SRSF1 stabilizes p53 via RPL5 and induces cellular senescence. Mol Cell 2013; 50: 56-66.

31. Tian B, Liu J, Liu B, Dong Y, Liu J, Song Y et al. p53 suppresses lung resistance-related protein expression through Y-box binding protein 1 in the MCF-7 breast tumor cell line. J Cell Physiol 2011; 226: 3433-3441.

32. Thoreen CC, Chantranupong L, Keys HR, Wang T, Gray NS, Sabatini DM. A unifying model for mTORC1-mediated regulation of mRNA translation. Nature 2012; 485: 109-113.

33. Feng Z. p53 regulation of the IGF-1/AKT/mTOR pathways and the endosomal compartment. Cold Spring Harb Perspect Biol 2010; 2: a001057.

34. Zhu X, Li Y, Shen H, Li H, Long L, Hui L et al. miR-137 restoration sensitizes multidrugresistant MCF-7/ADM cells to anticancer agents by targeting YB-1. Acta Biochim Biophys Sin (Shanghai) 2013; 45: 80-86.

35. Helwak A, Kudla G, Dudnakova T, Tollervey D. Mapping the human miRNA interactome by CLASH reveals frequent noncanonical binding. Cell 2013; 153: 654-665.

36. Das S, Anczuków O, Akerman M, Krainer AR. Oncogenic splicing factor SRSF1 is a critical transcriptional target of MYC. Cell Rep 2012; 1: 110-117.

37. Lasham A, Samuel W, Cao H, Patel R, Mehta R, Stern JL et al. YB-1, the E2F pathway, and regulation of tumor cell growth. J Natl Cancer Inst 2012; 104: 133-146.
38. Muñoz Ú, Puche JE, Hannivoort R, Lang UE, Cohen-Naftaly M, Friedman SL. Hepatocyte growth factor enhances alternative splicing of the Kruppel-like factor 6 (KLF6) tumor suppressor to promote growth through SRSF1. Mol Cancer Res 2012; 10: 1216-1227.

39. Weidensdorfer D, Stöhr N, Baude A, Lederer M, Köhn M, Schierhorn A et al. Control of c-myc mRNA stability by IGF2BP1-associated cytoplasmic RNPs. RNA 2009; 15 104-115.

40. Bugaut A, Balasubramanian S. 5'-UTR RNA G-quadruplexes: translation regulation and targeting. Nucleic Acids Res 2012; 40: 4727-4741.

41. Pichon X, Wilson LA, Stoneley M, Bastide A, King HA, Somers J et al. RNA binding protein RNA element interactions and the control of translation. Curr Protein Pept Sci 2012; 13: 294-304.

42. Dassi E, Re A, Leo S, Tebaldi T, Pasini L, Peroni D et al. AURA 2: empowering discovery of post-transcriptional networks. Translation 2014; 2: e27738.

43. Menendez D, Nguyen TA, Freudenberg JM, Mathew VJ, Anderson CW, Jothi R et al. Diverse stresses dramatically alter genome-wide p53 binding and transactivation landscape in human cancer cells. Nucleic Acids Res 2013; 41: 7286-7301.

44. Nicholson J, Neelagandan K, Huart AS, Ball K, Molloy MP, Hupp T. An iTRAQ proteomics screen reveals the effects of the MDM2 binding ligand nutlin-3 on cellular proteostasis. $J$ Proteome Res 2012; 11: 5464-5478.

45. Mazan-Mamczarz K, Hagner PR, Dai B, Wood WH, Zhang Y, Becker KG et al. Identification of transformation-related pathways in a breast epithelial cell model using a ribonomics approach. Cancer Res 2008; 68: 7730-7735.

46. Fernández-Miranda G, Méndez R. The CPEB-family of proteins, translational control in senescence and cancer. Ageing Res Rev 2012; 11: 460-472.

47. Novoa I, Gallego J, Ferreira PG, Mendez R. Mitotic cell-cycle progression is regulated by CPEB1 and CPEB4-dependent translational control. Nat Cell Biol 2010; 12: 447-456.

48. Fuller-Pace FV, Nicol SM. DEAD-box RNA helicases as transcription cofactors. Methods Enzymol 2012; 511: 347-367.

49. Das S, Fregoso OI, Krainer AR. A new path to oncogene-induced senescence: at the crossroads of splicing and translation. Cell Cycle 2013; 12: 1477-1479.

50. Leão M, Gomes S, Soares J, Bessa C, Maciel C, Ciribilli Y et al. Novel simplified yeastbased assays of regulators of p53-MDMX interaction and p53 transcriptional activity. FEBS J 2013; 280: 6498-6507.

51. Marcel V, Ghayad SE, Belin S, Therizols G, Morel AP, Solano-Gonzàlez E et al. p53 acts as a safeguard of translational control by regulating fibrillarin and rRNA methylation in cancer. Cancer Cell 2013; 24: 318-330.

52. Loayza-Puch F, Drost J, Rooijers K, Lopes R, Elkon R, Agami R. p53 induces transcriptional and translational programs to suppress cell proliferation and growth Genome Biol 2013; 14: R32.

53. Bisio A, Nasti S, Jordan JJ, Gargiulo S, Pastorino L, Provenzani A et al. Functional analysis of CDKN2A/p16INK4a 5'-UTR variants predisposing to melanoma. Hum Mol Genet 2010; 19: $1479-1491$.

54. Tebaldi T, Dessi E, Kostoska G, Viero G, Quattrone A. tRanslatome: an R/Bioconductor package to portrait translational control. Bioinformatics 2014; 30: 289-291.

55. Huang DW, Sherman BT, Lempicki RA. Systematic and integrative analysis of large gene lists using DAVID bioinformatics resources. Nat Protoc 2009; 4: 44-57.

56. Castello A, Fischer B, Eichelbaum K, Horos R, Beckmann BM, Strein C et al. Insights into RNA biology from an Atlas of mammalian mRNA-binding proteins. Cell 2012; 149 1393-1406.

57. Baltz AG, Munschauer M, Schwanhäusser B, Vasile A, Murakawa Y, Schueler M et al. The mRNA-bound proteome and its global occupancy profile on protein-coding transcripts. Mol Cell 2012; 46: 674-690.

58. Bustin SA, Benes V, Garson JA, Hellemans J, Huggett J, Kubista M et al. The MIQE guidelines: minimum information for publication of quantitative real-time PCR experiments. Clin Chem 2009; 55: 611-622.

59. Thoreen CC, Kang SA, Chang JW, Liu Q, Zhang J, Gao Y et al. An ATP-competitive mammalian target of rapamycin inhibitor reveals rapamycin-resistant functions of mTORC1. J Biol Chem 2009; 284: 8023-8032.

60. Denti MA, Rosa A, Sthandier O, De Angelis FG, Bozzoni I. A new vector, based on the Polll promoter of the U1 snRNA gene, for the expression of siRNAs in mammalian cells. Mol Ther 2004; 10: 191-199.

61. Ciribilli Y, Monti P, Bisio A, Nguyen HT, Ethayathulla AS, Ramos A et al. Transactivation specificity is conserved among p53 family proteins and depends on a response element sequence code. Nucleic Acids Res 2013; 41: 8637-8653. 